\title{
Phosphorylation of RS1 (RSC1A1) Steers Inhibition of Different Exocytotic Pathways for Glucose Transporter SGLT1 and Nucleoside Transporter CNT1, and an RS1-Derived Peptide Inhibits Glucose Absorption $\$$
}

\author{
Maike Veyhl-Wichmann, Alexandra Friedrich, Alexandra Vernaleken, Smriti Singh, \\ Helmut Kipp, Valentin Gorboulev, Thorsten Keller, Chakravarthi Chintalapati, \\ Rüdiger Pipkorn, Marçal Pastor-Anglada, Jürgen Groll, and Hermann Koepsell
}

Institute of Anatomy and Cell Biology, University Würzburg, Germany (M.V., A.V., H.Ki, V.G., C.C., H.Ko.); Department of Functional Materials in Medicine and Dentistry, University Hospital Würzburg, Germany (S.S., J.G.); Leibnitz Institute for Interactive Materials, Rheinisch-Westfälische Technische Hochschule, University Aachen, Germany (S.S.); German Cancer Research Center, Heidelberg, Germany (R.P.); Department of Biochemistry and Molecular Biology, Institute of Biomedicine, University Barcelona \& CIBER EHD, Barcelona, Spain (M.P.); and Department of Molecular Plant Physiology and Biophysics, Julius-von-Sachs-Institute, University Würzburg, Germany (A.F., T.K., H.Ko)

Received July 31, 2015; accepted October 9, 2015

\begin{abstract}
Cellular uptake adapts rapidly to physiologic demands by changing transporter abundance in the plasma membrane. The human gene RSC1A1 codes for a 67-kDa protein named RS1 that has been shown to induce downregulation of the sodium-Dglucose cotransporter 1 (SGLT1) and of the concentrative nucleoside transporter 1 (CNT1) in the plasma membrane by blocking exocytosis at the Golgi. Injecting RS1 fragments into Xenopus laevis oocytes expressing SGLT1 or CNT1 and measuring the expressed uptake of $\alpha$-methylglucoside or uridine 1 hour later, we identified a RS1 domain (RS1-Reg) containing multiple predicted phosphorylation sites that is responsible for this post-translational downregulation of SGLT1 and CNT1. Dependent on phosphorylation, RS1-Reg blocks the release of SGLT1-containing vesicles from the Golgi in a glucosedependent manner or glucose-independent release of CNT1containing vesicles. We showed that upregulation of SGLT1 in
\end{abstract}

the small intestine after glucose ingestion is promoted by glucose-dependent disinhibition of the RS1-Reg-blocked exocytotic pathway of SGLT1 between meals. Mimicking phosphorylation of RS1-Reg, we obtained a RS1-Reg variant that downregulates SGLT1 in the brush-border membrane at high luminal glucose concentration. Because RS1 mediates shortterm regulation of various transporters, we propose that the RS1-Reg-navigated transporter release from Golgi represents a basic regulatory mechanism of general importance, which implies the existence of receptor proteins that recognize different phosphorylated forms of RS1-Reg and of complex transporterspecific sorting in the trans-Golgi. RS1-Reg-derived peptides that downregulate SGLT1 at high intracellular glucose concentrations may be used for downregulation of glucose absorption in small intestine, which has been proposed as strategy for treatment of type 2 diabetes.

\section{Introduction}

Post-translational short-term regulations of plasma membrane transporters adapt transporter activities according to physiologic demands (Zhang et al., 2008; Kock et al., 2010;

This work was funded by the Deutsche Forschungsgemeinschaft [SFB 487/ C1, KO 872/5-1] and by the Ministry of Economy and Competitiveness [SAF2011-23660, SAF2014-52067-R].

M.V.-W., A.F., and A.V. contributed equally to this work.

dx.doi.org $/ 10.1124 / \mathrm{mol} .101162$

S This article has supplemental material available at molpharm. aspetjournals.com
Vina-Vilaseca et al., 2011; Gorboulev et al., 2012; Leto and Saltiel, 2012; Mühlfeld et al., 2012; Zhang et al., 2013). Physiologically important examples are insulin-induced recruitment of glucose transporter 4 (GLUT4) to the plasma membrane of adipocytes (Leto and Saltiel, 2012), protein kinase C-dependent endocytosis of organic anion transporter 1 (OAT1) (Zhang et al., 2008, 2013), and glucose-induced incorporation of the $\mathrm{Na}^{+}$-D-glucose cotransporter SGLT1 into brush-border membranes (BBMs) of small intestinal enterocytes (Gorboulev et al., 2012). We have observed that regulation of the release of SGLT1-containing vesicles from the

ABBREVIATIONS: AMG, $\alpha$-methyl-D-glucopyranoside; BBM, brush-border membrane; BFA, brefeldin A; BTXB, botulinum toxin B; CamK2, calmodulin kinase 2; CNT1, concentrative nucleoside transporter 1; DOG, 2-deoxyglucose; GLP-1, glucagon-like peptide 1; GLUT2, glucose transporter 2; GST, glutathione-S-transferase; IPTG, isopropyl-1-thio- $\beta$-D-galactopyranoside; NG, nanohydrogel; OCT, organic cation transporter; PCR, polymerase chain reaction; PMA, phorbol-12-myristate-13-acetate; QSP, Gln-Ser-Pro; $\mathrm{R}_{(\mathrm{CNT} 1)}$, proposed receptor protein for RS1-Reg that mediates regulation of CNT1; RS1, product of gene RSC1A1; RS1-Reg, NH2-terminal regulatory domain of RS1; $\mathrm{R}_{(\mathrm{SGLT1})}$, proposed receptor protein for RS1-Reg that mediates regulation of SGLT1; SGLT1, $\mathrm{Na}^{+}$-D-glucose cotransporter 1; TGN, trans-Golgi network. 
trans-Golgi network (TGN) contributes to short-term regulation of SGLT1 (Kroiss et al., 2006; Veyhl et al., 2006).

Human RS1 (hRS1) is encoded by the intron-less single-copy gene RSC1A1. This gene first appeared in mammals and encodes 67 - to $68-\mathrm{kDa}$ proteins with about $70 \%$ amino acid identity between species (Veyhl et al., 1993; Lambotte et al., 1996; Reinhardt et al., 1999; Osswald et al., 2005). RS1 is expressed in various tissues and cell types (Valentin et al., 2000; Korn et al., 2001; Osswald et al., 2005). In the renal epithelial cell line LLC-PK 1, RS1 protein was detected at the TGN, within the nucleus, and at the plasma membrane (Kroiss et al., 2006). At the TGN, RS1 was colocalized with SGLT1 (Kroiss et al., 2006). Evidence has been provided that RS1 participates in transcriptional and post-translational regulations of SGLT1. For example, we showed that RS1 is involved in the transcriptional upregulation of SGLT1 during the confluence of LLC-PK 1 cells (Korn et al., 2001). We identified a nuclear shuttling domain in the center of RS1 that steers nuclear migration of RS1 during confluence of LLC-PK $_{1}$ cells and observed that a COOH-terminal fragment of hRS1 is involved in the regulation of transcription (Filatova et al., 2009). To date, transcriptional regulation by RS1 has not been investigated in detail.

By expressing SGLT1 in oocytes of Xenopus laevis and injecting RS1 protein or a peptide motif of RS1, we characterized short-term post-translational effects on the abundance of SGLT1 in the plasma membrane (Veyhl et al., 2006; Vernaleken et al., 2007). We observed that short-term downregulation of hSGLT1 after injection of hRS1 protein was abolished when the Golgi was dissociated with brefeldin A (BFA) or when vesicle fusion with the plasma membrane was blocked with botulinum toxin B (BTXB) but was not influenced by inhibitors of endocytosis (Veyhl et al., 2006). The exocytotic pathway of hSGLT1 expressed in oocytes was also blocked after injection of nanomolar concentrations of the tripeptide motif Gln-Ser-Pro (QSP) in hRS1 (Vernaleken et al., 2007). The high affinity of QSP for downregulation of hSGLT1 suggests that QSP, and probably also hRS1, binds to an intracellular high-affinity receptor protein that mediates downregulation. Of note, we observed that the short-term downregulation of hSGLT1 by hRS1 or QSP was blunted after cellular injection of D-glucose or the nonmetabolized glucose analogs 2-deoxyglucose (2-DOG) or $\alpha$-methylglucoside (AMG) (Veyhl et al., 2006; Vernaleken et al., 2007). Because QSP is too small to form a glucose-binding site, the glucose-binding site mediating glucose dependence of QSP-mediated downregulation may be located at the receptor protein for RS1 or at a protein that is associated with the receptor protein. We have observed that hRS1 also downregulates the exocytotic pathway(s) of the human concentrative nucleoside transporters hCNT1, hCNT2, and hCNT3 (Errasti-Murugarren et al., 2012).

The aim of our present study was to find out whether hRS1 blocks a common exocytotic pathway for hSGLT1 and hCNT1 or whether different exocytotic pathways for SGLT1 and CNT1 exist that are addressed separately. We identified an $\mathrm{NH}_{2}$-terminal domain of hRS1 named hRS1-Reg that contains two QSP motifs within predicted phosphorylation sites, many additional predicted phosphorylation sites, and predicted binding sites for regulatory proteins. We showed that, dependent on phosphorylation of hRS1-Reg, either hSGLT1 was downregulated in a glucose-dependent manner, or hCNT1 was downregulated independently of glucose. Performing studies with RS1 knockout mice (Osswald et al., 2005) and wild-type mice, we provided evidence that glucose-dependent disinhibition of RS1-induced post-translational downregulation of SGLT1 in small intestine is responsible for upregulation of SGLT1 after glucose-rich meals.

\section{Materials and Methods}

Animals. C57BL/6 wild-type mice and $R s 1^{-1-}$ mice on C57BL/6 background (Osswald et al., 2005) were kept in temperaturecontrolled environment with 12 -hour light/dark cycles. The mice were provided with standard chow and water ad libitum (Altronin 1324; Altromin Spezialfutter GmbH, Lage, Germany). Animals were handled in compliance with the guidelines of the University of Würzburg and German laws.

Reagents. $\alpha$-Methyl-D- $\left[{ }^{14} \mathrm{C}\right]$ glucopyranoside $\quad\left(\left[{ }^{14} \mathrm{C}\right] \mathrm{AMG}\right) \quad(11.1$ $\mathrm{GBq} / \mathrm{mmol})$ and $\left[5-{ }^{3} \mathrm{H}\right]$ uridine $(0.91 \mathrm{TBq} / \mathrm{mmol})$ were obtained from American Labeled Chemical Inc. (St. Louis, MO). GlutathioneSepharose was purchased from GE Healthcare Europe GmbH and KN93 from Merck-Millipore GmbH (Darmstadt, Germany). Span 80, and Tween 80 were obtained from Sigma-Aldrich GmbH (Taufkirchen, Germany). Soluene-350 was obtained from PerkinElmer Inc. (Waltham MA). TAT-peptide (Torchilin, 2008) was delivered by Peptides International (Louisville, Kentucky). Other chemicals were purchased as described (Pipkorn et al., 2002; Keller et al., 2005; Vernaleken et al., 2007).

Preparation of Nanohydrogels with Coupled Peptides. Nanohydrogels (NGs) were prepared from inversed miniemulsions as described (Groll et al., 2009). Inversed miniemulsions were prepared from $2.5 \mathrm{ml}$ of hexane containing $50.3 \mathrm{mg}$ of Span 80 and $16.7 \mathrm{mg}$ of Tween 80 and $0.4 \mathrm{ml}$ of $20 \mathrm{mM}$ Hepes pH 7.4 containing $100 \mathrm{mg}$ of thiol-functionalized linear poly(glycidol) polymer (Groll et al., 2009), $0.5 \mathrm{mg}$ TAT-peptide (CGRLLRRQRRR) (Torchilin, 2008), and $0.28 \mathrm{mg}$ of cysteine-terminated mRS1-Reg polypeptide. For preparation of control NG, mRS1-Reg polypeptide was omitted. For generation of the inversed miniemulsions, the organic and aqueous phases were combined, stirred, and sonicated at $0^{\circ} \mathrm{C}$. Then $60 \mu \mathrm{l}$ of water containing $1 \mathrm{M} \mathrm{H}_{2} \mathrm{O}_{2}$ was added for the formation of disulfide bridges. The mixtures were sonicated for 60 seconds and incubated for 25 minutes at room temperature. Oxidation was quenched by acidification, and the NGs were separated by centrifugation. The aqueous layers containing the NGs were separated and washed four times with a tetrahydrofuran/water $(20 \% / 80 \%)$ mixture to remove surfactants and unreacted polymer. Remaining organic solvents and acid were removed by dialysis against water. The suspensions containing $50 \mathrm{mg} / \mathrm{ml}$ NGs loaded with $0.225 \mathrm{mg}$ of mRS1-Reg polypeptides were stored for up to 2 weeks at $4^{\circ} \mathrm{C}$.

Cloning. For coexpression with hSGLT1 in oocytes, hRS1 cDNA variants coding for the hRS1 fragments shown in Fig. 1 were generated on the basis of wild-type hRS1 in vector pRSSP (pRSSP/ hRS1) (Busch et al., 1996). They were cloned into ApaI and XhoI sites of pRSSP. To synthesize variants coding for amino acids 1-251, 1-183, $1-140$, and $1-111$ of $\mathrm{hRS} 1$, polymerase chain reaction (PCR) was performed using pRSSP plasmid-specific forward primer and reverse primers at the corresponding positions of hRS1, which contained a stop codon and XhoI restriction site. The amplificates were digested with ApaI and XhoI and inserted into the pRSSP vector. To prepare cDNA variants coding for hRS1 fragments 50-183, 20-50, 30-50, and 3040 , PCR was performed with hRS1-derived forward primers containing the ApaI adaptor and the initiation codon. The hRS1derived reverse primers contained the XhoI adaptor. Amplificates were digested with ApaI and XhoI and inserted into pRSSP vector.

At the beginning of the study, oocyte experiments with injected peptides were performed with glutathione-S-transferase (GST) fusion proteins of hRS1-Reg and of hRS1-Reg mutants [hRS1-Reg(S45A), hRS1-Reg(S45E), hRS1-Reg(S83A), hRS1-Reg(S83E)]. The peptides 


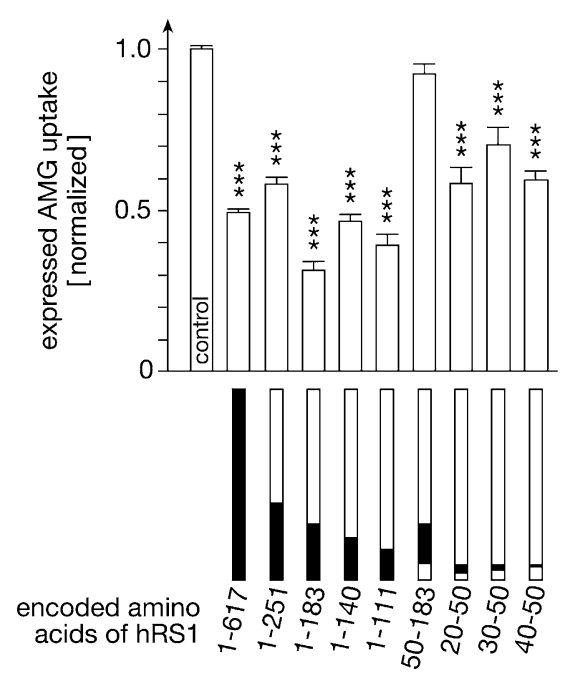

Fig. 1. Identification of an $\mathrm{NH}_{2}$-terminal hRS1 cRNA fragment that reduces the expression of hSGLT1-mediated AMG transport after coexpression with hSGLT1. hSGLT1 cRNA alone (control) or hSGLT1 cRNA together with cRNAs encoding total hRS1 (amino acids 1-617) or hRS1 fragments were injected into oocytes. Two days later, uptake of $50 \mu \mathrm{M}$ AMG was measured in cRNA injected oocytes and in noninjected oocytes, and the uptake mediated by hSGLT1 was calculated. Mean values \pm S.E. of 16-27 cRNA injected oocytes from two or three independent experiments that were corrected for uptake in oocytes without cRNA injection are shown. $* * * P<0.001$ for difference from control calculated by ANOVA with post hoc Tukey comparison.

were linked to the $\mathrm{C}$ terminus of GST using the vector pGEX5X-3 (GE Healthcare Europe GmbH, Freiburg, Germany) for expression in Escherichia coli. Later experiments were performed with uncoupled hRS1-Reg, hRS1-Reg mutants [hRS1-Reg(S20A), hRS1-Reg(S20E)], mRS1-Reg, and mRS1-Reg mutants [mRS1-Reg(S19A), mRS1-Reg (S19E)] expressed in vector pET21a. The uncoupled variants contained an $\mathrm{NH}_{2}$-terminal cysteine that was used for coupling to NGs.

hRS1 (Lambotte et al., 1996) and mRS1 (GenBank accession no. Y11917) were used as templates to synthesize hRS1-Reg comprising amino acids Ser16-Gln98 of hRS1 and mRS1-Reg comprising Pro15His92 of mRS1-Reg by PCR (Fig. 3). Mutants were generated by applying the PCR overlap extension method (Ho et al., 1989).

For expression of GST fusion proteins, PCR of hRS1-Reg, hRS1-Reg (S45A), hRS1-Reg(S45E), hRS1-Reg(S83A), and hRS1-Reg(S83E) was performed using hRS1-derived primers containing BamHI and SalI sites. The amplification products were cut with BamHI and SalI and cloned into the vector pGEX5X-3 in the open reading frame with the $\mathrm{N}$-terminal GST.

For expression of uncoupled hRS1-Reg, hRS1-Reg mutants [hRS1Reg(S20A), hRS1-Reg(S20E)], PCR was performed using the forward primer $5^{\prime}$ TATACATATGTGCTCTTCAGGACAGAGTCCTG $3^{\prime}$ and the reverse primer 5' GTCTCGAGCTGCATAGGCATAGCTGG $3^{\prime}$. For expression of uncoupled mRS1-Reg, mRS1-Reg(S19A), and mRS1-Reg (S19E), the forward primer 5' GGTACATATGTGTCCTTCAGGGCA GAGTCCTG $3^{\prime}$ and the reverse primer 5' GTCTCGAGATGGAAAGGCATGGCTAGACTC 3 ' were used. The forward primers were provided with an NdeI recognition site (underlined), an initiation codon (bold), and a codon for cysteine (italic). The reverse primers contained a XhoI recognition site (underlined). The PCR amplificates were cut with NdeI and XhoI and cloned into the vector pET21a (Novagen, Darmstadt, Germany) in the open reading frame with the C-terminal His-Tag.

Expression and Purification of GST-Fusion Proteins. E. coli bacteria (strain BL21 STAR, Live Technologies, Darmstadt, Germany) were transfected with pGEX5X-3 plasmids and grown to $50 \%$ maximal density. After induction by $1 \mathrm{mM}$ isopropyl-1-thio- $\beta$-Dgalactopyranoside (IPTG) bacteria were grown for 3 hours at $30^{\circ} \mathrm{C}$, pelleted by 15 -minute centrifugation at $6,000 \mathrm{~g}$, washed, and suspended in $20 \mathrm{mM}$ Hepes, pH 7.5 containing $150 \mathrm{mM} \mathrm{NaCl}$. Bacteria were lysed by sonication at $4^{\circ} \mathrm{C}$, and cellular debris was removed by 1-hour centrifugation at $100,000 \mathrm{~g}$. For protein purification, $0.5 \mathrm{ml}$ of glutathione-Sepharose (GE Healthcare, Munich, Germany) was added to $10 \mathrm{ml}$ of lysate and incubated for 1 hour at $4^{\circ} \mathrm{C}$. The suspension was poured into an empty gravity flow column, washed with $25 \mathrm{ml}$ of $20 \mathrm{mM}$ Hepes, $\mathrm{pH} 7.5$, containing $150 \mathrm{mM} \mathrm{NaCl}$, and the GST-fusion proteins were eluted with $2.5 \mathrm{ml}$ of $20 \mathrm{mM}$ Hepes, $\mathrm{pH} 7.5$, containing $150 \mathrm{mM}$ $\mathrm{NaCl}$ and $20 \mathrm{mM}$ glutathione. Fractions containing purified protein were pooled and dialyzed against K-Ori buffer ( $5 \mathrm{mM}$ MOPS, pH 7.4, $103 \mathrm{mM} \mathrm{KCl}, 1 \mathrm{mM} \mathrm{MgCl} 2$ ).

Expression and Purification of Uncoupled hRS1-Reg, mRS1-Reg, and Mutants. $E$. coli bacteria (strain BL21) were transformed with pET21a plasmids containing His-tagged hRS1-Reg or mRS1-Reg and grown to mid-log phase. Protein expression was induced by IPTG, and bacteria were grown for 3 hours at $30^{\circ} \mathrm{C}$. After 15-minute centrifugation at $6000 \mathrm{~g}$, bacteria were washed, suspended in $20 \mathrm{mM}$ Tris- $\mathrm{HCl}, \mathrm{pH} 8.0$, containing $500 \mathrm{mM} \mathrm{NaCl}$ and $50 \mathrm{mM}$ imidazole, lysed by sonication at $4^{\circ} \mathrm{C}$, and cellular debris was removed by 1 -hour centrifugation at $100,000 \mathrm{~g}$. For protein purification, the supernatants were mixed with $\mathrm{Ni}^{2+}$-NTA-Agarose (Qiagen, Hilden, Germany), incubated for 1 hour under rotation, and poured into an empty gravity flow column. After extensive washing with $20 \mathrm{mM}$ Tris$\mathrm{HCl}, \mathrm{pH} 8.0$ containing $500 \mathrm{mM} \mathrm{NaCl}$ and $50 \mathrm{mM}$ imidazole, protein was eluted with the same buffer containing $500 \mathrm{mM}$ imidazole. Fractions containing purified protein were pooled and dialyzed against $20 \mathrm{mM}$ Hepes, $\mathrm{pH} 7.5$, containing $150 \mathrm{mM} \mathrm{NaCl}$ (for coupling to NG) or against K-Ori buffer (for injection into oocytes).

cRNA Synthesis. For cRNA synthesis human SGLT1 (hSGLT1) in vector pBSII SK (Hediger and Rhoads, 1994), human hCNT1 (hCNT1) in vector pBSII KS (Errasti-Murugarren et al., 2012), human RS1 (hRS1) in vector pRSSP (Busch et al., 1996), and hRS1-fragments in pRSSP were used. To prepare sense cRNAs, the respective purified plasmids were linearized with EcoRI (hSGLT1), MluI (hRS1), or XbaI (hCNT1). The $\mathrm{m}^{7} \mathrm{G}\left(5^{\prime}\right) \mathrm{G}$-capped sense cRNAs were synthesized using T3 polymerase (hSGLT1, hCNT1) or SP6 polymerase (fragments of hRS1). cRNAs were prepared using the mMESSAGE mMachine kit (Ambion Life Technologies, Austin, TX) using sodium acetate precipitation. cRNA concentrations were estimated from ethidium bromide-stained agarose gels using polynucleotide marker as standards (Gründemann and Koepsell, 1994).

Expression of Transporters and hRS1 Fragments in Oocytes of Xenopus laevis. Mature female Xenopus laevis were anesthetized by immersion in water containing $0.1 \% 3$-aminobenzoic acid ethyl ester. After partial ovariectomy, stage V or VI oocytes were treated overnight with $1 \mathrm{mg} \mathrm{ml}^{-1}$ collagenase I in Ori buffer $(5 \mathrm{mM}$ HEPES, $\mathrm{pH}$ 7.6, $110 \mathrm{mM} \mathrm{NaCl}, 3 \mathrm{mM} \mathrm{KCl}, 1 \mathrm{mM} \mathrm{MgCl}_{2}$, and $2 \mathrm{mM}$ $\mathrm{CaCl}_{2}$ ). The oocytes were washed with $\mathrm{Ca}^{2+}$-free Ori buffer and kept at $16^{\circ} \mathrm{C}$ in modified Barth's solution [15 mM HEPES, pH 7.6, $88 \mathrm{mM}$ $\left.\mathrm{NaCl}, 1 \mathrm{mM} \mathrm{KCl}, 0.3 \mathrm{mM} \mathrm{Ca}\left(\mathrm{NO}_{3}\right)_{2}, 0.4 \mathrm{mM} \mathrm{CaCl}_{2}, 0.8 \mathrm{mM} \mathrm{MgSO}_{4}\right]$ containing $12.5 \mu \mathrm{M}$ gentamycin. Selected oocytes were injected with $50 \mathrm{nl}$ of water containing cRNAs (2.5 ng of hSGLT1, $0.5 \mathrm{ng}$ of hCNT1, and/or $7.5 \mathrm{ng}$ of fragments of hRS1). For expression, oocytes were incubated for 2 days at $16^{\circ} \mathrm{C}$ in modified Barth's solution with gentamycin. Noninjected control oocytes were incubated in parallel.

Injection of Peptides, AMG, and Biochemicals into Oocytes. Two days after injection of transporter cRNAs into oocytes, peptides AMG, BFA, BTXB, and/or PKC inhibitors were injected into oocytes 1 hour before uptake measurements were performed. After injection of peptides, some oocytes were incubated for 2 minutes with $1 \mu \mathrm{M}$ phorbol-12-myristate-13-acetate (PMA) for PKC stimulation and others for 1 hour with $10 \mu \mathrm{M}$ of KN93 for inhibition of calmodulin kinase 2 (CamK2) (Sumi et al., 1991). We injected $40 \mathrm{nl}$ of K-Ori (5 mM MOPS, $\mathrm{pH} 7.4,103 \mathrm{mM} \mathrm{KCl}, 1 \mathrm{mM} \mathrm{MgCl}_{2}$ ) containing different amounts of RS1-derived peptides, 100 pmol AMG, 5 pmol BFA, 2 ng $\mathrm{BTXB}$, and 0.12 pmol calphostin C. Intracellular concentrations of injected compounds were estimated by assuming an internal distribution volume of $0.4 \mu \mathrm{l}$ (Zeuthen et al., 2002). 
Measurements of AMG or Uridine Uptake in Oocytes. Determination of hSGLT1-mediated AMG uptake was performed by correcting AMG uptake in hSGLT1-expressing oocytes for AMG uptake measured in non-cRNA-injected oocytes, which were handled in parallel. Oocytes were incubated for 20 minutes at room temperature in Ori buffer containing $50 \mu \mathrm{M}$ AMG traced with $\left[{ }^{14} \mathrm{C}\right] \mathrm{AMG}$. Thereafter, the oocytes were washed four times with ice-cold Ori buffer containing $1 \mathrm{mM}$ phlorizin. hCNT1-mediated uridine uptake was determined by measuring the difference of uridine uptake between oocytes in which hCNT1 was expressed by cRNA-injected and noncRNA-injected oocytes. Oocytes were incubated for 20 minutes at room temperature with Ori buffer containing $5 \mu \mathrm{M}$ uridine traced with $\left[{ }^{3} \mathrm{H}\right]$ uridine and washed four times with ice-cold Ori buffer. Single oocytes were solubilized in 5\% (w/v) SDS and analyzed for radioactivity by scintillation counting.

Gavage of Mice with Nanohydrogels. To investigate in vivo effects, male $R s 1^{-/-}$mice or wild-type mice fasted overnight with free access to water underwent gavage with $200 \mu \mathrm{l}$ of water (adjusted with $\mathrm{HCl}$ to $\mathrm{pH} 5.5$ ) containing $10 \mathrm{mg}$ of unloaded NGs or $10 \mathrm{mg}$ of NGs loaded with $45 \mu \mathrm{g}$ of mRS1-Reg mutant; 3 hours after gavage, mice were sacrificed. The small intestines were removed, perfused at room temperature with Krebs-Ringer buffer ( $25 \mathrm{mM}$ HEPES, $108 \mathrm{mM} \mathrm{NaCl}$, $4.8 \mathrm{mM} \mathrm{KCl}, 1.2 \mathrm{mM} \mathrm{KH}_{2} \mathrm{PO}_{4}, 1.2 \mathrm{mM} \mathrm{CaCl}_{2}, \mathrm{pH}$ 7.4), and everted using a steel rod. The jejunum was cut into eight 1-cm-long segments, and phlorizin-inhibited AMG uptake or sodium-dependent uridine uptake was measured. The procedure was performed in accordance with the guidelines of the University Würzburg.

Measurements of Phlorizin-Inhibited AMG Uptake and Sodium-Dependent Uridine Uptake in the Small Intestine. For measurement of SGLT1-mediated glucose uptake, the everted segments of mouse jejunum were incubated for 2 minutes at $37^{\circ} \mathrm{C}$ with Krebs-Ringer buffer containing $10 \mu \mathrm{M}$ of AMG or $1 \mathrm{mM}$ AMG containing tracer amounts of $\left[{ }^{14} \mathrm{C}\right] \mathrm{AMG}$. The incubations were performed in the absence or presence of $0.2 \mathrm{mM}$ or $1 \mathrm{mM}$ phlorizin for measurements of $10 \mu \mathrm{M}$ AMG uptake and $1 \mathrm{mM}$ AMG uptake, respectively. Because glucose-dependent upregulation of SGLT1 in the small intestine occurs within 2 minutes (Fig. 8, D and E), measurements with $1 \mathrm{mM}$ AMG record the upregulated situation at high intracellular glucose. After incubation with radioactively labeled AMG, uptake was stopped by transferring the segments into ice-cold Krebs-Ringer buffer containing $0.2 \mathrm{mM}$ phlorizin. For measurement of uridine uptake by sodium-uridine cotransporters, everted segments of mouse jejunum were incubated for 2 minutes at $37^{\circ} \mathrm{C}$ with $\mathrm{Krebs}-$ Ringer buffer containing $1 \mu \mathrm{M}$ uridine or Krebs-Ringer buffer in which $\mathrm{Na}^{+}$was replaced by $\mathrm{K}^{+}$plus $1 \mu \mathrm{M}$ uridine. Uptake was stopped by transferring the segments into ice-cold Krebs-Ringer buffer without sodium. For analysis of AMG and uridine uptake, the jejunal segments were dissolved in $0.5 \mathrm{ml}$ of Soluene-350. Radioactivity was analyzed by liquid scintillation counting. Phlorizin-inhibited AMG uptake and $\mathrm{Na}^{+}$-dependent uridine uptake per centimeter of length were calculated using four $1 \mathrm{~cm}$-segments per experimental condition.

Preparation of Brush-Border Membrane Vesicles from Small Intestine. The BBM vesicles were isolated by magnesium precipitation followed by differential centrifugation. Small intestines of three mice were homogenized at $0^{\circ} \mathrm{C}$ in $35 \mathrm{ml}$ of $2 \mathrm{mM}$ Hepes/Tris, $\mathrm{pH}$ 7.1, containing $100 \mathrm{mM}$ mannitol. $1 \mathrm{M} \mathrm{MgCl}_{2}$ solution was added to a concentration of $10 \mathrm{mM}$, and the suspension was kept on ice for 20 minutes and centrifuged for 15 minutes at $3000 \mathrm{~g}$. The pellet containing nuclei and mitochondria was discarded, and the supernatant was centrifuged for 30 minutes at $27,000 \mathrm{~g}$. The resulting pellet was suspended in $35 \mathrm{ml}$ of vesicle buffer (20 mM Hepes/Tris, $\mathrm{pH} 7.4$, $100 \mathrm{mM}$ mannitol) and centrifuged for 30 minutes at $27,000 \mathrm{~g}$. The obtained pellet containing the BBM vesicles was suspended in $0.3 \mathrm{ml}$ of vesicle buffer, snap-frozen in liquid nitrogen, and stored at $-70^{\circ} \mathrm{C}$ until use. In BBM vesicles isolated from wild-type mice, the activity of alkaline phosphatase was enriched $12 \pm 2$ fold (S.D., $n=10$ ) compared with the homogenates. In BBM vesicles prepared from $R s 1^{-1-}$ mice, the same enrichment of alkaline phosphatase activity was obtained.
Measurement of SGLT1-Mediated AMG Uptake into BBM Vesicles. The BBM vesicles were thawed in a water bath, and the phlorizin-inhibitable uptake of $\mathrm{AMG}$ was measured at $22^{\circ} \mathrm{C}$ using the rapid filtration technique (Hopfer et al., 1973). BBM vesicles were incubated for 10 seconds with vesicle buffer containing $0.1 \mathrm{mM} \mathrm{AMG}$ traced with $\left[{ }^{14} \mathrm{C}\right] \mathrm{AMG}$ plus either $100 \mathrm{mM}$ NaSCN or $100 \mathrm{mM}$ NaSCN and $0.2 \mathrm{mM}$ phlorizin. Uptake was stopped with ice-cold vesicle buffer, which contained $0.2 \mathrm{mM}$ phlorizin. The vesicles were washed on nitrocellulose filters using the same solution. The radioactivity on the filters was measured, and phlorizin inhibitable uptake was calculated.

Estimation of SGLT1 and Glucose Transporter 2 Protein in BBM Vesicles. The BBM vesicles were separated by SDS-PAGE, transferred to polyvinylidene difluoride membrane, and probed with antibody against mouse SGLT1 or antibody against human GLUT2 that cross-reacts with mouse glucose transporter 2 (GLUT2) (Gorboulev et al., 2012). Quantification of immunostaining was performed by densitometry (Gorboulev et al., 2012).

Statistics. Uptake measurements indicated in the graphs are presented as mean \pm S.E. In experiments with oocytes, each mean value was calculated from uptake measurements in 16 to 27 individual transporter-expressing oocytes from two or three independent experiments, which were corrected for uptake of oocytes without transporter expression. The inhibition curves shown in the graphs were obtained by fitting the Hill equation to the compiled data sets. The downregulation $\mathrm{EC}_{50}$ values are presented as mean \pm S.D. They were calculated from two or three $\mathrm{EC}_{50}$ values obtained by fitting the Hill equation to dose-response curves of individual experiments. Uptake rates of phlorizin-inhibited AMG uptake into BBM vesicles were calculated from three independent experiments. In each experiment, three uptake measurements were performed in the absence of phlorizin and corrected for uptake measured in the presence of phlorizin. Uptake rates measured in intestinal mucosa were calculated from measurements in four to seven animals. For each animal, the uptake rate was calculated from eight measurements using two experimental conditions (AMG uptake without and with phlorizin, uridine uptake in the presence and absence of sodium). When three or more groups of data were compared, the significance of differences was determined by analysis of variance (ANOVA) using post hoc Tukey comparison. The significance of the differences between the two groups was determined by Student's $t$ test. $\mathrm{P}<0.05$ was considered significant.

\section{Results}

Identification of a hRS1 Domain that Blocks the Exocytotic Pathway of hSGLT1. Previously, we have described that injection of $0.1 \mu \mathrm{M}$ QSP into hSGLT1-expressing $X$. laevis oocytes induced a downregulation of hSGLT1mediated glucose uptake by about $40 \%$ (Vernaleken et al., 2007). Performing coexpression of hSGLT1 with various $\mathrm{NH}_{2}-$ terminal hRS1-cRNA fragments in $X$. laevis oocytes, we observed that a cRNA fragment encoding the amino acids IKPSDSDRIEP decreased hSGLT1 expressed uptake of $50 \mu \mathrm{M}$ AMG to a similar degree as QSP (Fig. 1). To further define and functionally characterize the active peptide motif, we performed short-term inhibition experiments with IKPSDSDRIEP and fragments of this peptide (Fig. 2). We expressed hSGLT1 in oocytes by cRNA injection and incubation for 2 days, injected peptides, and measured expressed uptake of $50 \mu \mathrm{M}$ AMG 1 hour later. Because we had determined previously that injection of RS1 protein and QSP into hSGLT1-expressing oocytes decreased the amount of SGLT1 in the plasma membrane without altering the uptake kinetics of AMG (Veyhl et al., 2006; Vernaleken et al., 2007), the observed changes in AMG uptake were interpreted to indicate changes of hSGLT1 


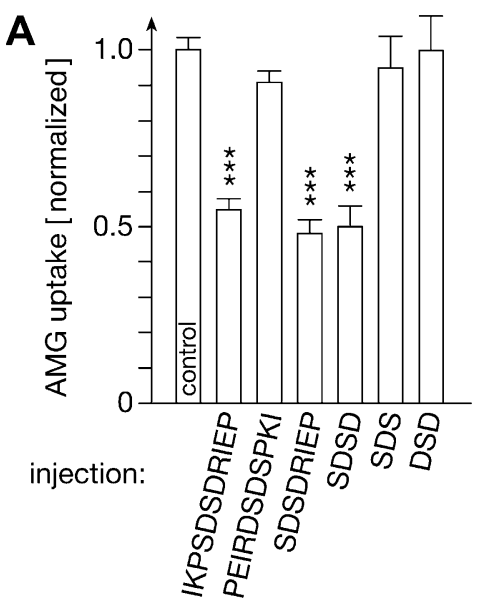

B
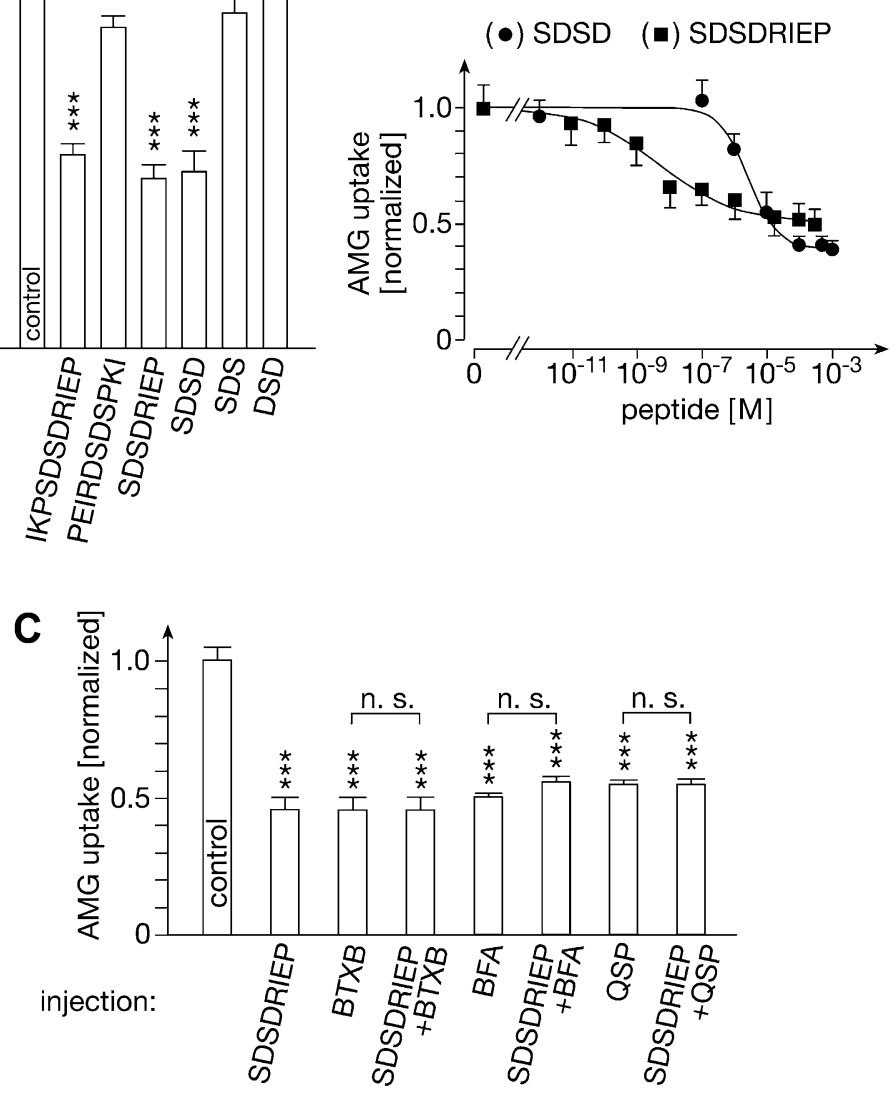

Fig. 2. Identification and characterization of an $\mathrm{NH}_{2-}$ terminal peptide motif in hRS1 that induces short-term posttranslational decrease of hSGLT1 expressed AMG transport. Oocytes were injected with hSGLT1-cRNA and incubated 2 days for expression. Thereafter, oocytes were injected with potassium-rich buffer without peptides (controls in $\mathrm{A}$ and $\mathrm{C}$, zero peptide concentration in $\mathrm{B}$ ), or potassium-rich buffer containing different amounts of the peptides, $2 \mathrm{ng}$ BTXB and/or 5 pmol BFA. After 1 hour incubation, hSGLT1-expressed uptake of $50 \mu \mathrm{M}$ AMG was measured. (A) Identification of the shortest active peptide motif; 40 pmol of the peptides were injected, leading to intracellular peptide concentration of $\sim 100 \mu \mathrm{M}$. (B) Concentration dependence of SDSD and SDSDRIEP for downregulation of hSGLT1-mediated AMG uptake. The intracellular concentrations of the peptides are indicated. (C) SDSDRIEP and QSP downregulate the BFA sensitive exocytotic pathway of hSGLT1 in a nonadditive way; $40 \mathrm{pmol}$ of the peptides was injected. Mean values \pm S.E. of 24-27 cRNA injected oocytes from three independent experiments corrected for uptake in oocytes without cRNA injection are shown. Curves were obtained by fitting the Hill equation to the compiled data sets. For downregulation of AMG uptake by SDSD and SDSDRIEP, $\mathrm{EC}_{50}$ values of $2.64 \pm 0.47 \mu \mathrm{M}$ and $4.7 \pm 0.4 \mathrm{nM}$ were calculated (mean \pm S.D. calculated from $\mathrm{EC}_{50}$ determinations of three individual experiments; $P<0.05$ for difference). $* * * P<0.001$ for difference from control calculated by ANOVA with post hoc Tukey comparison. abundance in the plasma membrane. Figure 2A shows that also the octapeptide SDSDRIEP and the tetrapeptide SDSD downregulate hSGLT1; however, the affinity of SDSDRIEP was more than 500-fold higher compared with SDSD (Fig. 2B). As observed after injection of total hRS1 protein or QSP (Veyhl et al., 2006; Vernaleken et al., 2007), the inhibition of hSGLT1mediated AMG uptake by SDSDRIEP was abolished when exocytosis was blocked by BTXB or when the Golgi was disintegrated by BFA (Fig. 2C). Since the effects of SDSDRIEP and QSP on the expression of hSGLT1 were not additive (Fig. 2C), both peptides act on the same exocytotic pathway. After injection of $100 \mathrm{pmol}$ AMG into the oocytes, leading to a cytosolic concentration of $\sim 0.25 \mathrm{mM}$, the inhibitory effect of QSP was abolished (Supplemental Fig. 1A), whereas the $\mathrm{EC}_{50}$ for downregulation by SDSDRIEP was increased from $4.7 \pm 0.4 \mathrm{nM}$ to $504 \pm 64 \mathrm{nM}$ (mean \pm S.D. from three experiments; $P<0.001$ for difference) (Supplemental Fig. 1B). Previously, we had verified that in control oocytes without peptide injection, AMG injection did not influence hSGLT1-mediated AMG uptake (Vernaleken et al., 2007).

SDSDRIEP and two QSP motifs are located within amino acids 16-98 of hRS1 (Fig. 3), suggesting that this fragment, named hRS1-Reg, comprises the post-translational regulatory domain of hRS1. hRS1-Reg contains 16 serine residues and one threonine residue. For all these residues, phosphorylation is predicted (program GPS version $2.1 \mathrm{http} / /$ gps.biocuckoo. org). In hRS1-Reg five binding motifs for Src homology (SH) proteins and two binding motifs for protein 14-3-3 are also predicted (program Minimotif miner http://mnm.engr.uconn. edu/MNM/SMSSearchServlet).
The characteristics of hRS1-Reg were similar to QSP and SDSDRIEP. When hRS1-Reg was injected into oocytes expressing hSGLT1, AMG uptake was downregulated to the same level as after injection of BFA (Fig. 4A). Downregulation by BFA and hRS1-Reg were not additive (Fig. 4A). The $\mathrm{EC}_{50}$ for downregulation by hRS1-Reg $(68 \pm 3.7 \mathrm{nM}$, mean \pm S.D. from three experiments) (Fig. 4B) was much higher compared with QSP $(0.17 \pm 0.03 \mathrm{nM}$, mean \pm S.D. from three experiments; $P<0.001$ for difference) (Supplemental Fig. 1A) and SDSDRIEP $(4.7 \pm 0.4 \mathrm{nM}$, mean $\pm \mathrm{SD}$ from three experiments, $P<0.001$ for difference to hRS1-Reg) (Fig. 2B). After injection of $100 \mathrm{pmol} \mathrm{AMG}$, the $\mathrm{EC}_{50}$ for inhibition of AMG uptake by hRS1-Reg was increased from $68 \pm 3.7 \mathrm{nM}$ to $540 \pm 5.0 \mathrm{nM}$ (mean \pm S.D. from three experiments; $P<$ 0.001 for difference) (Fig. 4B). These data suggest that QSP and SDSDRIEP are binding motifs within hRS1-Reg that bind to a receptor protein-associated with the TGN. We

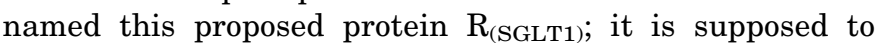
modulate the release of hSGLT1-containing vesicles in a glucose-dependent way.

hRS1-Reg Phosphorylation by PKC or Calmodulin Kinase 2 Changes Affinity for Downregulation of hSGLT1. We determined the affinity of hRS1-Reg for downregulation of hSGLT1-mediated AMG uptake after stimulation of $\mathrm{PKC}$ with phorbol-12-myristate-13-acetate (PMA), after inhibition of $\mathrm{PKC}$ with calphostin $\mathrm{C}$ and after inhibition of CamK2 with KN93 (Fig. 5, A, B, and D). The $\mathrm{EC}_{50}$ for downregulation by hRS1-Reg was not changed by calphostin $\mathrm{C}$ but decreased by PMA from $68 \pm 3.7 \mathrm{nM}$ to $5.1 \pm 0.3 \mathrm{nM}$ (mean \pm S.D. from three experiments; $P<0.001$ for the 


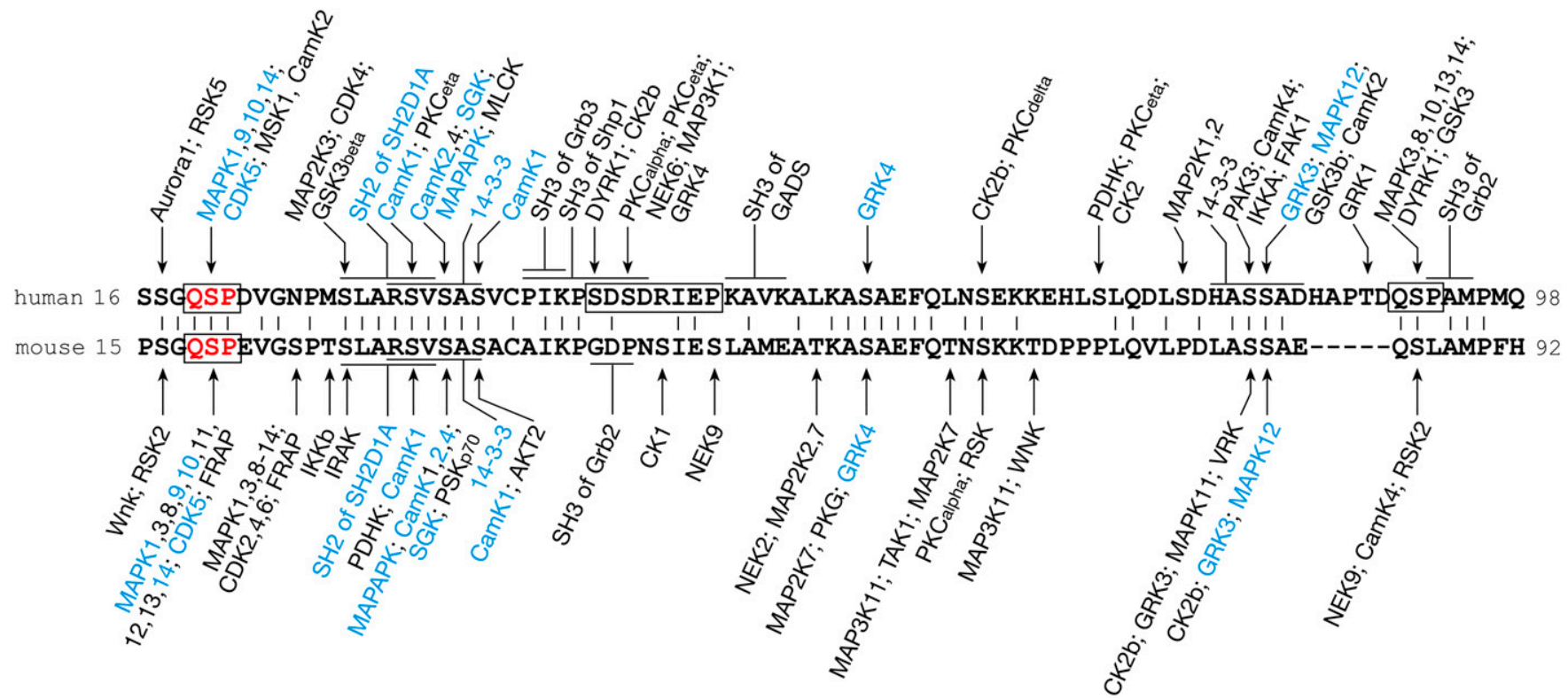

Fig. 3. Amino acid sequences of hRS1-Reg and mRS1-Reg with predicted phosphorylation sites and protein-binding sites. Identical amino acids are connected by lines. QSP and SDSDRIEP are boxed, and the conserved QSP motif is indicated in red. Predicted phoshorylation and protein binding sites, which are conserved between human and mouse, are shown in blue.

difference) (Fig. 5A). The higher degree of maximal downregulation observed in the presence of PMA suggests an additional PMA effect on hSGLT1 expression. KN93 decreased the $\mathrm{EC}_{50}$ for inhibition of hRS1-Reg for downregulation of hSGLT1 dramatically from $68 \pm 3.7 \mathrm{nM}$ to $112 \pm 4.3 \mathrm{fM}$ (mean \pm S.D. from three experiments; $P<0.001$ for the difference) (Fig. 5B). We investigated whether phosphorylation of the predicted PKC-dependent phosphorylation site at serine 45 in the SDSDRIEP motif is critical for the PMA effect on affinity. After blocking phosphorylation by alanine replacement [hRS1-Reg(S45A)], the affinity was not changed significantly (Fig. 5, C and E). After mimicking phosphorylation by replacement with glutamate [hRS1-Reg(S45E)], however, the $\mathrm{EC}_{50}$ for inhibition was decreased to the same level as with hRS1-Reg in the presence of PMA $(4.5 \pm 1.9 \mathrm{nM}$ compared with $5.1 \pm 0.3 \mathrm{nM}$, mean \pm S.D. from three experiments) (Fig. 5C). The data indicate that PKC increases the affinity of hRS1-Reg for downregulation of hSGLT1 by phosphorylation of Ser45.

CamK2-dependent phosphorylation is predicted for Ser20, Ser34, and Ser83 of human RS1 (Fig. 3). When we prevented phosphorylation at Ser83 by replacement with alanine [hRS1$\operatorname{Reg}(\mathrm{S} 83 \mathrm{~A})]$, we obtained a similar $\mathrm{EC}_{50}$ value for downregulation of hSGLT1-mediated AMG uptake as with hRS1-Reg in the presence of KN93 $(93 \pm 5.7 \mathrm{fM}$ vs. $112 \pm 4.3 \mathrm{fM}$, mean \pm S.D. from three experiments) (Fig. 5B). After exchange of Ser83 by glutamate $[\mathrm{hRS} 1-\operatorname{Reg}(\mathrm{S} 83 \mathrm{E})]$, the $\mathrm{EC}_{50}$ value was similar to hRS1-Reg without inhibition of CamK2 $(55 \pm 0.23 \mathrm{nM}$ compared with $68 \pm 3.7 \mathrm{nM}$, mean \pm S.D. from three experiments) (Fig. 5B). The data suggest that in oocytes hRS1-Reg is phosphorylated at serine 83 and that the affinity increase observed after blockage of CamK2 is due mainly to dephosphorylated Ser83.

Next we investigated whether the AMG-induced affinity decrease in hRS1-Reg is influenced by PKC or CamK2dependent phosphorylation. After injection of $100 \mathrm{pmol}$
AMG, a similar decrease in hRS1-Reg affinity was observed in the absence and presence of calphostin C (Fig. 5D). The drastic decrease in $\mathrm{EC}_{50}$ for downregulation of hRS1-Reg by KN93 was reversed after injection of $100 \mathrm{pmol} A M G(112 \pm 4.3 \mathrm{fM}$ vs. $117 \pm 44 \mathrm{nM})($ mean \pm S.D. from three experiments; $P<0.001$ for the difference) (Fig. 5D). To investigate whether AMGdependent phosphorylation of Ser83 causes the AMG-induced affinity decrease, we determined the effect of AMG on the affinity of hRS1-Reg(S83A). After injection of $100 \mathrm{pmol}$ AMG into oocytes, the $\mathrm{EC}_{50}$ of hRS1-Reg(S83A) for downregulation

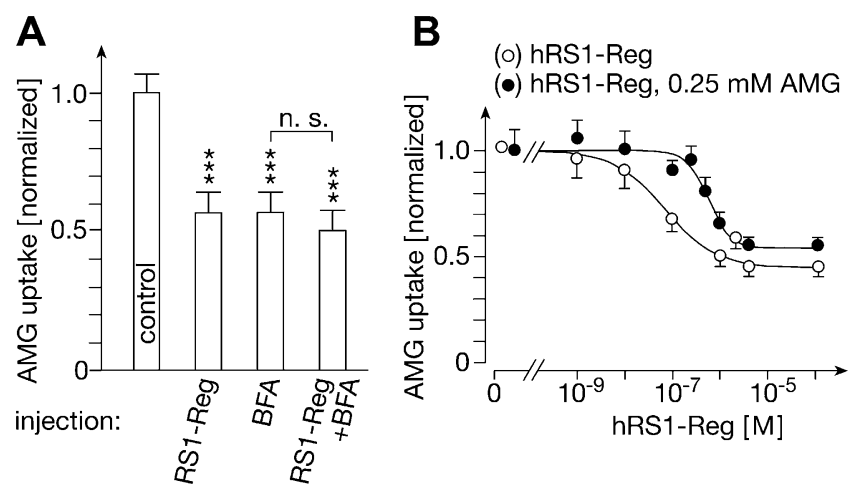

Fig. 4. Characterization of hSGLT1 regulation by hRS1-Reg. hSGLT1 was expressed in oocytes by cRNA injection and 2 days of incubation. Thereafter, oocytes were injected with potassium-rich (control in A, zero peptide concentration in $\mathrm{B}$ ) or potassium-rich buffer containing different amounts of hRS1-Reg, 2 ng BTXB, 5 pmol BFA, and/or 100 pmol AMG. After 1-hour incubation, hSGLT1-expressed AMG uptake was measured. (A) hRS1-Reg downregulates the BFA-sensitive exocytotic pathway of hSGLT1. (B) The affinity of hRS1-Reg for downregulation of hSGLT1 is decreased after AMG injection. Mean values \pm S.E. of 24-27 peptideinjected hSGLT1-expressing oocytes from three independent experiments are presented. Values were corrected for uptake in oocytes in which hSGLT1 was not expressed. The curves were obtained by fitting the Hill equation to the compiled data sets. $* * * P<0.001$ for significance of difference from control tested by ANOVA with post hoc Tukey comparison. 

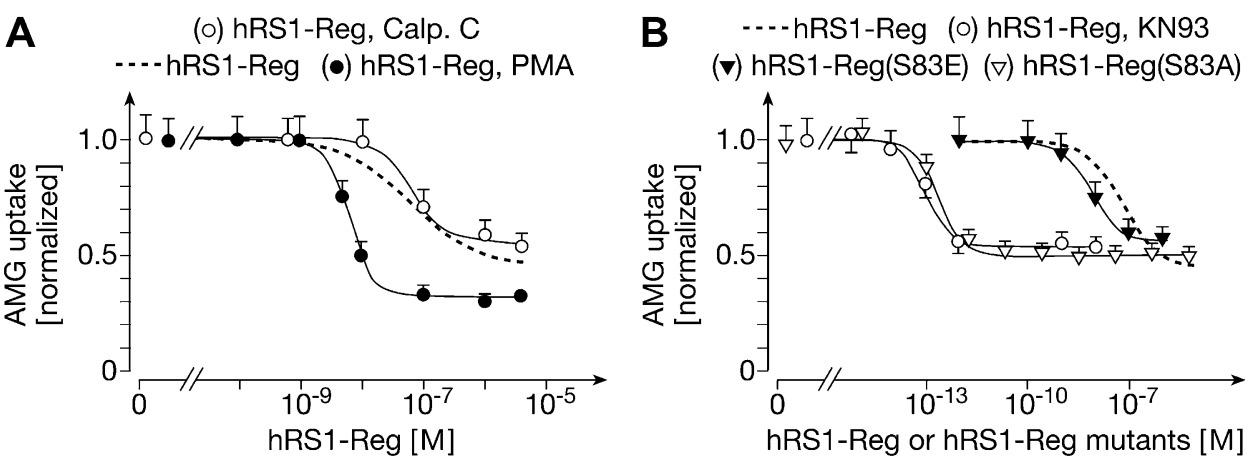

Fig. 5. Effects of phosphorylation of hRS1-Reg at serines 45 and 83 on downregulation of hSGLT1 at low and high intracellular glucose. hSGLT1-expressing oocytes were injected with different amounts of hRS1-Reg or hRS1-Reg mutants. In some experiments, $0.12 \mathrm{pmol}$ calphostin $\mathrm{C}$ and/or 100 pmol AMG were injected together with the peptides. In other experiments, the oocytes were incubated for 2 minutes with $1 \mu \mathrm{M}$ phorbol12-myristate-13-acetate (PMA) or 1 hour with $10 \mu \mathrm{M} \mathrm{KN} 93$ after the peptide injection. One hour after peptide injection, hSGLT1-mediated AMG uptake was measured. Inhibition curves indicated by broken lines are shown for comparison. (A) The affinity of hRS1-Reg increased when PKC was stimulated by PMA. (B) The affinity of hRS1-Reg was increased when CamK2 was inhibited by KN93 or when phosphorylation of Ser83 was prevented by alanine replacement. (C) Affinity of hRS1Reg increased when phosphorylation of Ser45 was mimicked by glutamate replacement. (D) Injection of AMG into the oocytes $(0.25 \mathrm{mM}$ intracellular concentration) decreased the affinity of hRS1-Reg after inhibition of PKC with calphostin $\mathrm{C}$ and after inhibition of CamK2 with KN93 (open bars without AMG injection, closed bars with AMG injection). (E) Effects of AMG injection on the affinities of hRS1-Reg variants with mutations of serine 45 or 83 (open bars without AMG injection, closed bars with AMG injection). The AMG-induced affinity decrease was prevented after mutations of Ser45 and after replacement of Ser83 by glutamate. (A-C) Mean values \pm S.E. of 16 27 cRNA injected oocytes from two or three independent experiments corrected for uptake in oocytes without cRNA injection are shown. The curves were obtained by fitting the Hill equation to the compiled data sets. $(\mathrm{D}, \mathrm{E}) \mathrm{Mean} \mathrm{EC}_{50}$ values \pm S.D. are shown that were calculated from individual experiments. The numbers of experiments are indicated in parentheses. Significances of differences were calculated by ANOVA with post hoc Tukey comparison. $* P<0.05 ; * * P<$ $0.01 ; * * * P<0.001 ;$ n.s., not significant

of hSGLT1 was increased from $93 \pm 5.7 \mathrm{fM}$ to $455 \pm 74 \mathrm{nM}$ (mean \pm S.D. from three experiments; $P<0.001$ for difference) (Fig. 5E). This excludes that AMG-dependent affinity decrease is mediated via phosphorylation of Ser83.

Mimicking Phosphorylation of $\mathrm{Ser}$ in the $\mathrm{NH}_{2}$ Terminal QSP Motif of hRS1-Reg or mRS1-Reg Increases Affinity for Downregulation of SGLT1 at High Intracelluar Glucose. The $\mathrm{NH}_{2}$-terminal QSP in RS1-Reg is conserved between human and mouse (Fig. 3). In both species, phosphorylation of this serine is predicted by MAP kinases 1, 9, 10, 14, and cyclin-dependent kinase 5. Mass spectrometry of purified hRS1 protein that had been overexpressed in Sf9 insect cells revealed that serine in the $\mathrm{NH}_{2}-$ terminal QSP (Ser20) was phosphorylated (A. Friedrich, C. Otto, P. Reddy Rikkala, M. Veyhl-Wichmann, Y. Reinders, C.F.Jurowich, H. Koepsell, unpublished data). We investigated whether the affinity for downregulation of hSGLT1 by hRS1Reg was altered when Ser20 in hRS1-Reg was exchanged by alanine [hRS1-Reg(S20A)] or glutamate [(hRS1-Reg(S20E)] (Fig. 6, A and E). The $\mathrm{EC}_{50}$ of hRS1-Reg(S20A) for downregulation of hSGLT1 was similar to hRS1-Reg, suggesting that Ser20 of hRS1-Reg is not phosphorylated in the oocytes. 
A -..-hRS1-Reg (o) hRS1-Reg(S20E) (ㅁ) hRS1-Reg(S20A)
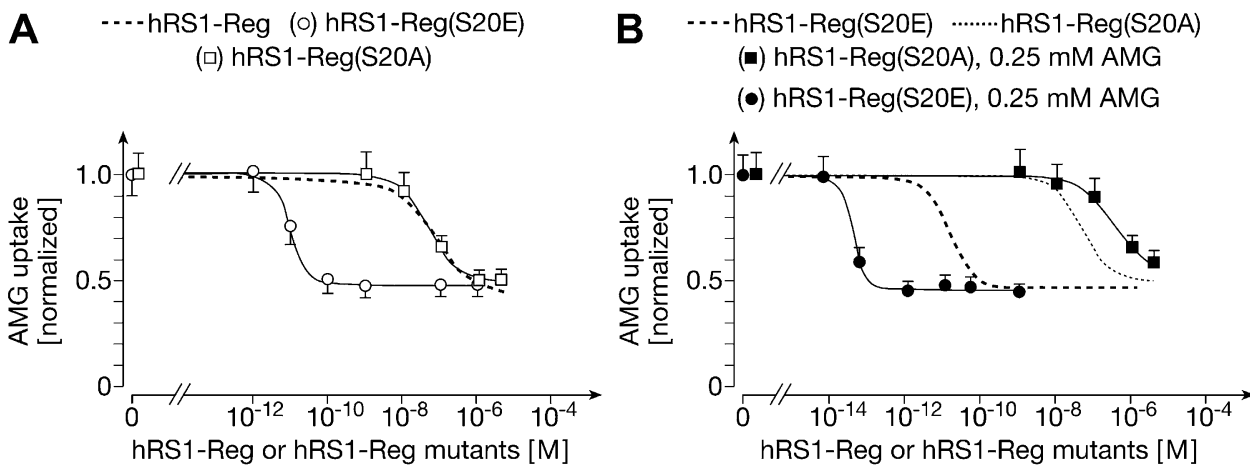

C

(o) mRS1-Reg(S19E)

() $\mathrm{mRS} 1-\operatorname{Reg}(\mathrm{S} 19 \mathrm{~A})$

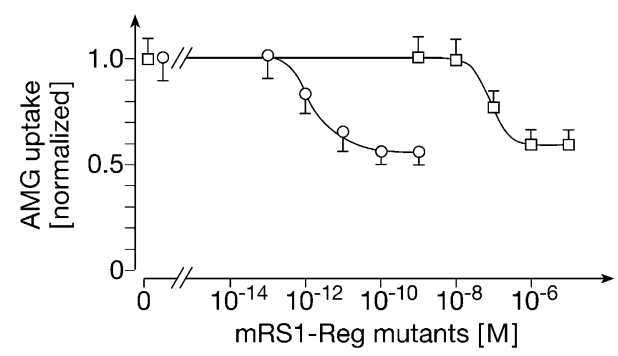

D

...-mRS1-Reg(S19E) ……mRS1-Reg(S19A)

(•) mRS1-Reg(S19E), 0.25 mM AMG

(घ) mRS1-Reg(S19A), $0.25 \mathrm{mM} \mathrm{AMG}$
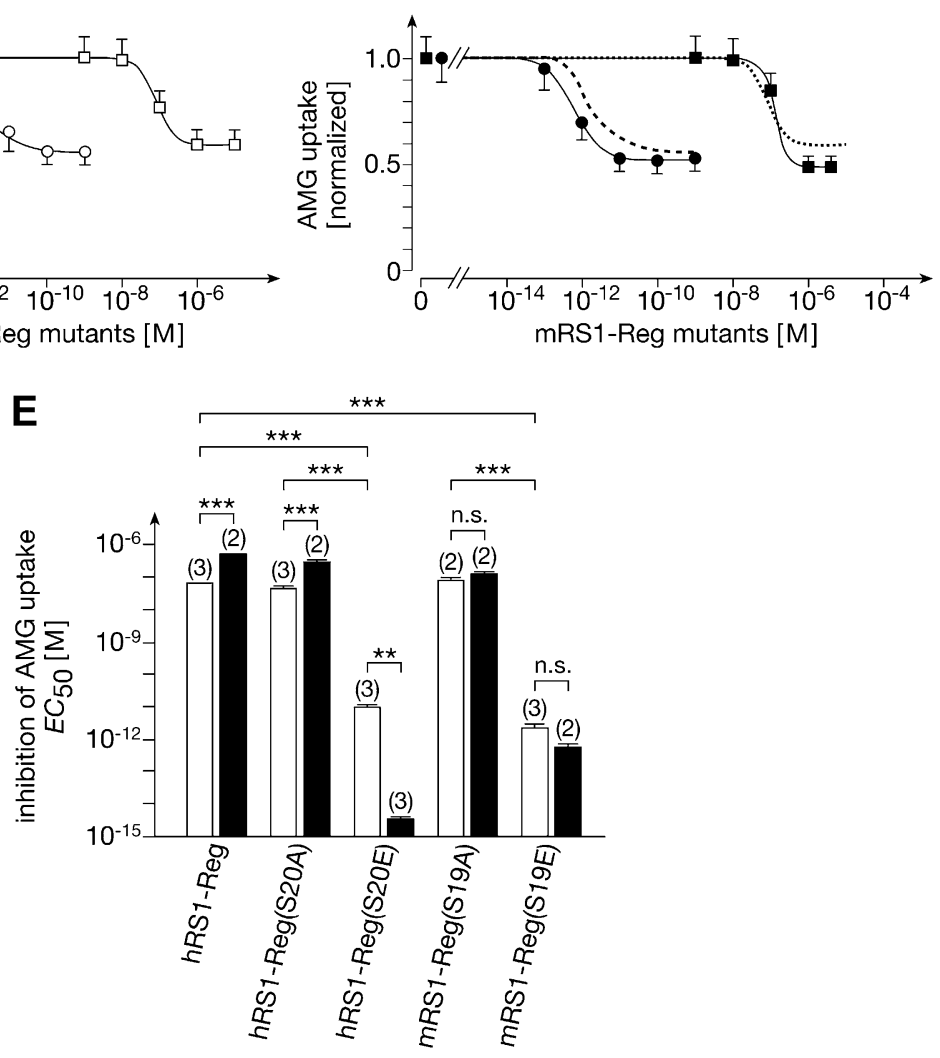

Fig. 6. Effects of phosphorylation of the QSP motif in hRS1-Reg and mRS1-Reg on downregulation of hSGLT1 at low and high intracellular glucose. hSGLT1expressing oocytes were injected with different amounts of hRS1-Reg, hRS1Reg mutants, mRS1-Reg mutants, and/or 100 pmol AMG. One hour after peptide injection, hSGLT1-mediated AMG uptake was measured. (A) Mimicking phosphorylation of Ser20 in hRS1 (hRS1-Reg(S20E)) increased the affinity dramatically. (B) After injection of AMG, the affinity of hRS1-Reg(S20A) was decreased, whereas the affinity of hRS1-Reg(S20E) was increased. (C) Mimicking phosphorylation of Ser19 in mRS1-Reg (mRS1-Reg(S19E)) increased the affinity dramatically. (D) After injection of AMG the affinities of mRS1-Reg(S19A) and mRS1-Reg(S19E) were not changed significantly (see also Fig. $6 \mathrm{E}$ ). (E) Comparison of $\mathrm{EC}_{50}$ values for downregulation of hSGLT1-mediated AMG uptake without AMG injection (open bars) and with injection of AMG (closed bars). The experiments were performed, and the data are presented as in Fig. 5 . $* * P<0.01 ; * * * P<0.001$ ANOVA with post hoc Tukey comparison.
In contrast, the $\mathrm{EC}_{50}$ of $\mathrm{hRS} 1-\mathrm{Reg}(\mathrm{S} 20 \mathrm{E})$ was much lower compared with hRS1-Reg $(19 \pm 0.23$ pM vs. $68 \pm 3.7 \mathrm{nM}$, mean \pm S.D. for three experiments; $P<0.001$ for the difference). After injection of $100 \mathrm{pmol}$, AMG the $\mathrm{EC}_{50}$ of hRS1-Reg(S20A) was increased from $48 \pm 7.7 \mathrm{nM}$ to $338 \pm 19 \mathrm{nM}$, mean \pm S.D. from two and three experiments; $P<0.001$ for the difference) (Fig. 6, B and E). Surprisingly, the $\mathrm{EC}_{50}$ of hRS1-Reg(S20E) was decreased after injection of AMG $(19 \pm 0.23$ pM vs. $33 \pm 1.0 \mathrm{fM}$, mean \pm S.D. from three experiments; $P<0.01$ for the difference) (Fig. 6 , $\mathrm{B}$ and $\mathrm{E}$ ).

Investigating regulation of SGLT1 by murine RS1-Reg (mRS1-Reg), we determined the effects of serine replacement in the QSP motif of mRS1-Reg. For downregulation of hSGLT1expressed AMG uptake in oocytes by mRS1-Reg(S19A) and mRS1-Reg(S19E), respective $\mathrm{EC}_{50}$ values of $8.5 \pm 2.4 \mathrm{nM}$ and $2.0 \pm 1.4 \mathrm{pM}$ were obtained (mean \pm S.D. from two or three experiments; $P<0.01$ for the difference) (Fig. 6, C and E). After injection of 100 pmol AMG into the oocytes, similar $\mathrm{EC}_{50}$ values were obtained (Fig. 6, D and E). The data indicate that phosphorylation of serine in the conserved QSP motif of RS1Reg increases the affinity for downregulation of SGLT1 in different species.

hRS1-Reg Downregulates Sodium Nucleoside Cotransporter hCNT1 and hSGLT1 by Different Pathways. Previously, we described post-translational downregulation of the human nucleoside transporters hCNT1-3 by hRS1 that was abolished when the Golgi was disintegrated by BFA (Errasti-Murugarren et al., 2012). To determine whether hRS1-Reg addresses a common or different exocytotic pathway(s) for downregulation of hSGLT1 versus hCNT1, we also performed a detailed characterization of hRS1-Regmediated downregulation of hCNT1. For hRS1-Reg-mediated 
downregulation of hCNT1-mediated uridine uptake (oocytes without $\mathrm{AMG}$ injection), a higher $\mathrm{EC}_{50}$ value was obtained compared with hSGLT1-mediated AMG uptake (Fig. 7A)
$(125 \pm 13 \mathrm{nM}$ vs. $68 \pm 3.7 \mathrm{nM}$, mean \pm S.D. of three experiments; $P<0.01$ for the difference). hCNT1-mediated uridine uptake was downregulated by SDSDRIEP but not by
A

(o) hRS1-Reg

(•) hRS1-Reg, $0.25 \mathrm{mM} \mathrm{AMG}$

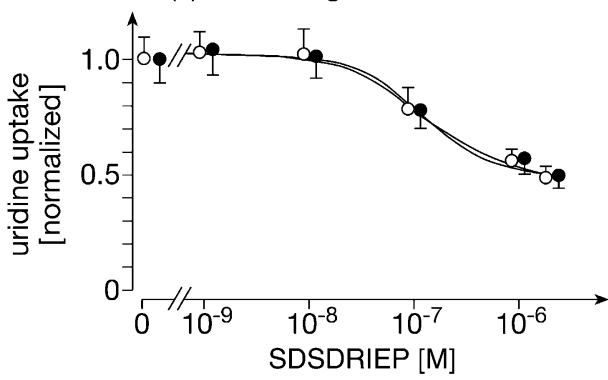

C

(o) SDSDRIEP

(•) hRS1-Reg, 0.25 mM AMG

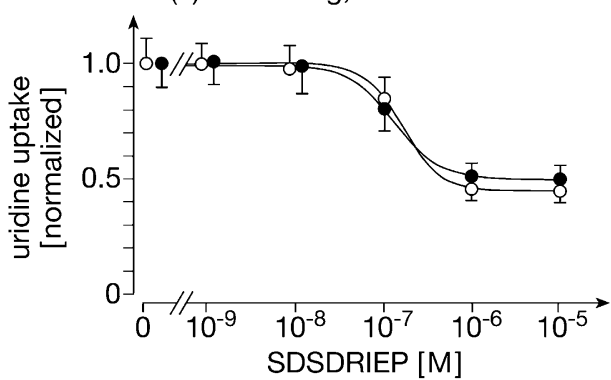

$\mathbf{E}$ -..-hRS1-Reg (o) hRS1-Reg(S83A) (•) hRS1-Reg(S83E)

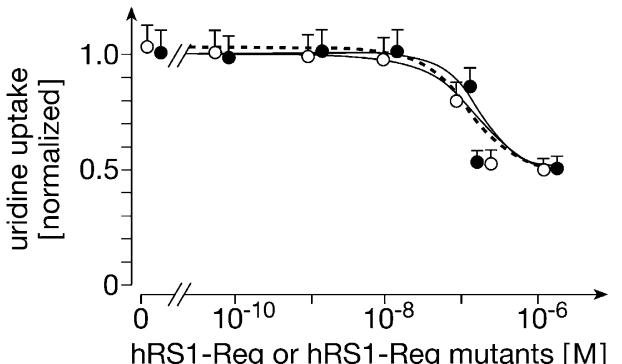

B

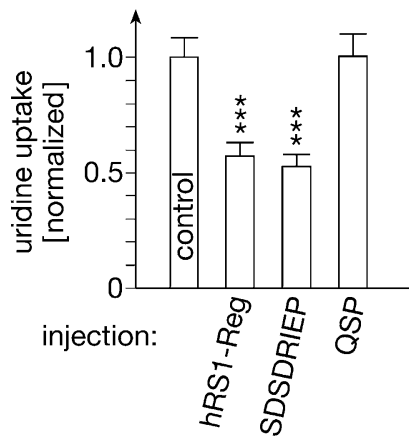

D $\quad \cdots \cdot h R S 1-\operatorname{Reg}$ (ㅁ) hRS1-Reg(S45A)

(घ) hRS1-Reg(S45E)

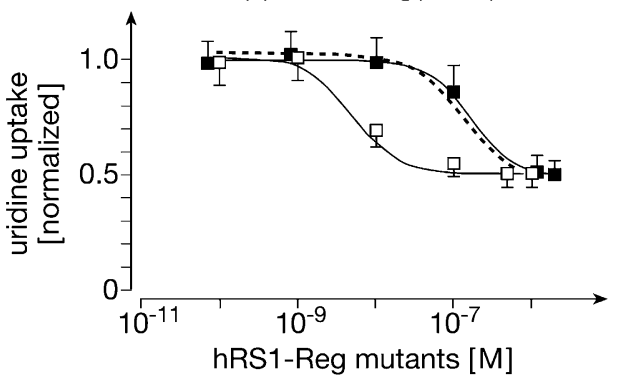

F $\quad \cdots \cdot h R S 1-R e g(0) h R S 1-R e g(S 20 A)$

(•) hRS1-Reg(S20E)

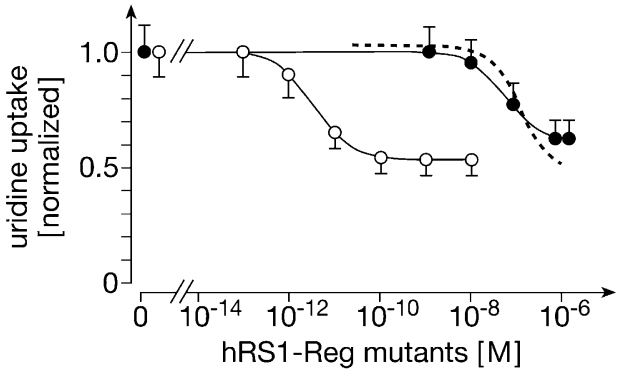

Fig. 7. Downregulation of hCNT1 by hRS1-Reg at low and high intracellular glucose, by SDSDRIEP and QSP, and by mutants of hRS1-Reg and mRS1-Reg. hCNT1-expressing oocytes were injected with hRS1-Reg, SDSDRIEP, QSP, hRS1Reg mutants, mRS1-Reg mutants, and/or 100 pmol AMG, and sodium dependent uptake of $5 \mu \mathrm{M}$ uridine mediated by hCNT1 was measured 1 hour later. (A) Affinity of hRS1-Reg for downregulation of hCNT1 was independent of intracellular glucose. (B) hCNT1 was downregulated by SDSDRIEP but not by QSP; 40 pmol of hRS1-Reg, SDSDRIEP or QSP were injected, leading to an intracellular concentration of $\sim 100 \mu \mathrm{M}$. (C) Downregulation of hCNT1 by SDSDRIEP was independent of intracellular glucose. (D) Affinity of hRS1-Reg for downregulation increased when Ser45 was replaced by alanine but remained unchanged when it was replaced by glutamate. (E) Replacement of Ser83 in hRS1-Reg by alanine or glutamate did not change affinity for downregulation of hCNT1. (F) Replacement of Ser20 in the first QSP motif of hRS1-Reg by alanine increased affinity for downregulation of hCNT1 dramatically, whereas replacement by glutamate had only a small effect. (G) Comparison of $\mathrm{EC}_{50}$ values for downregulation of hCNT1-mediated uridine uptake by hRS1-Reg, mutants of hRS1-Reg, and mutants of mRS1-Reg. $\mathrm{EC}_{50}$ values for downregulation were calculated from independent experiments by fitting the Hill equation to dose-response curves. Mean values \pm S.D. are shown and the number of independent experiments are indicated in parentheses. Significances of differences were calculated by ANOVA with post hoc Tukey comparison. ${ }^{\bullet} P<0.01$; $\bullet P<0.001$ for difference to hRS1-Reg; *** $P<0.001$ for indicated comparison. 
QSP (Fig. 7B), and the $\mathrm{EC}_{50}$ for downregulation of hCNT1 by SDSDRIEP was much higher compared with the $\mathrm{EC}_{50}$ for downregulation of hSGLT1 by SDSDRIEP $(165 \pm 8.8 \mathrm{nM}$ vs. $4.7 \pm 0.37 \mathrm{nM}$, mean \pm S.D. of three experiments; $P<0.001$ for difference) (Figs. 2B and 7C). In contrast to downregulation of hSGLT1 by hRS1-Reg or SDSDRIEP, the downregulation of hCNT1 was independent of intracellular glucose (compare Fig. 4B and Supplemental Fig. 1B with Fig. 7, A and C). The data indicate that the post-translational downregulation of hSGLT1 and hCNT1 at the TGN occurs via independent exocytotic pathways. This finding suggests that different receptor proteins for $\mathrm{hRS} 1$ are involved, a glucose-dependent receptor protein addressing hSGLT1 $\left(\mathrm{R}_{(\mathrm{SGLT1}}\right)$ and a glucoseindependent receptor protein addressing hCNT1, which we named $\mathrm{R}_{(\mathrm{CNT} 1)}$.

RS1-Reg Phosphorylation Determines Whether SGLT1 or hCNT1 Is Downregulated. The EC $_{50}$ of hRS1Reg for the downregulation of uridine uptake mediated by hCNT1 in oocytes $(125 \pm 13 \mathrm{nM})$ was decreased in the presence of calphostin $\mathrm{C}$ to $5.0 \pm 1.9 \mathrm{nM}$ (mean \pm S.D. of two or three experiments; $P<0.01$ for the difference). This effect is in contrast to the downregulation of hSGLT1mediated AMG uptake in oocytes without AMG injection, which was not altered by calphostin $\mathrm{C}$ (Fig. 5, A and D). To determine the role of Ser45, we compared the affinities of hRS1-Reg(S45A) versus hRS1-Reg(S45E) for the downregulation of hCNT1. Whereas replacement of Ser45 by glutamate decreased the $\mathrm{EC}_{50}$ for the downregulation of hSGLT1 (Fig. 5, $\mathrm{C}$ and $\mathrm{E})$, the $\mathrm{EC}_{50}$ value for downregulation of hCNT1 was decreased when Ser45 was replaced by alanine [hRS1-Reg $125 \pm 13 \mathrm{nM}, \mathrm{hRS} 1-\operatorname{Reg}(\mathrm{S} 45 \mathrm{~A}) 8.5 \pm 1.4 \mathrm{nM}, \mathrm{hRS} 1-\operatorname{Reg}(\mathrm{S} 45 \mathrm{E})$ $173 \pm 18 \mathrm{nM}$; mean \pm S.D. from three experiments; $P<0.001$ for the difference between hRS1-Reg(S45A) and hRS1-Reg] (Fig. 7, D and G). The data suggest that PKC dependent phosphorylation of Ser45 is involved in navigating downregulation of hSGLT1 versus hCNT1.

When Ser83 in hRS1-Reg was replaced by alanine or glutamate, the affinity for downregulation of hCNT1mediated uridine uptake was not altered significantly (Fig. 7, $\mathrm{E}$ and $\mathrm{G}$ ). This finding is in contrast to the regulation of hSGLT1, where the affinity of hRS1-Reg(S83A) was much higher compared with hRS1Reg(S83E) (Fig. 5, B and E).

Different effects on the affinity for downregulation of hSGLT1 versus hCNT1 were also observed after mimicking or preventing the phosphorylation of Ser20 in hRS1-Reg. Whereas the $\mathrm{EC}_{50}$ of hRS1-Reg(S20E) for downregulation of hCNT1 was $39 \%$ lower than hRS1-Reg wild-type $(77 \pm 16 \mathrm{nM}$ vs. $125 \pm 13 \mathrm{nM}$, mean \pm S.D. of three experiments; $P<0.01$ for the difference) (Fig. $7, \mathrm{~F}$ and $\mathrm{G}$ ), the $\mathrm{EC}_{50}$ value for downregulation of hCNT1 by hRS1-Reg(S20A) was more than three orders of magnitude lower compared with hRS1-Reg wild-type $(3.8 \pm 1.4 \mathrm{pM}$ vs. $125 \pm 13 \mathrm{nM}$, mean \pm S.D. of three experiments; $P<0.001$ for the difference) (Fig. $7, \mathrm{~F}$ and $\mathrm{G}$ ). On the other hand, the $\mathrm{EC}_{50}$ value for downregulation of hSGLT1 by hRS1-Reg(S20E) was more than three orders of magnitude lower compared with hRS1-Reg wild-type, whereas the $\mathrm{IC}_{50}$ for the downregulation of hSGLT1 by hRS1-Reg(S20A) was similar to that of hRS1-Reg (Fig. 6, A and E). Similar differences were observed for mutations in the QSP motif of mRS1Reg. The $\mathrm{EC}_{50}$ for downregulation of hCNT1-mediated uridine uptake by mRS1-Reg(S19A) was more than two orders of magnitude lower compared with that by mRS1-Reg(S19E)
$(53 \pm 2.9$ pM vs. $122 \pm 1.0 \mathrm{nM}$, mean \pm S.D. of two experiments; $P<0.001$ for the difference) (Fig. 7G). By contrast, the $\mathrm{EC}_{50}$ for downregulation of hSGLT1-mediated AMG uptake by mRS1Reg(S19A) was more than two orders of magnitude higher compared with that by mRS1-Reg(S19E) $(8.5 \pm 2.4 \mathrm{nM}$ and $2.0 \pm 1.4 \mathrm{pM}$, mean \pm S.D. of three experiments; $P<0.01$ for difference) (Fig. 6, C and E).

Involvement of RS1 in Upregulation of Glucose Absorption in the Small Intestine after a GlucoseRich Meal. Previously, we showed that SGLT1 in the BBM of enterocytes is rate-limiting for small intestinal glucose absorption (Gorboulev et al., 2012). We also reported that SGLT1 in the BBM of murine small intestine was upregulated after gavage with glucose. To determine whether RS1 is involved in this glucose-dependent upregulation of SGLT1, we performed gavage on male wild-type mice and $R s 1^{-1-}$ mice using D-glucose, sacrificed the animals 30 minutes later, and isolated BBM vesicles from jejunum. We measured phlorizin-inhibited AMG uptake into the BBM vesicles (Fig. 8A) and quantified SGLT1 (Fig. 8B) and facilitative glucose transporter 2 (GLUT2) (Fig. $8 \mathrm{C}$ ) in the BBM in immunostained Western blots. In the jejunum of $R s 1^{-/-}$mice, the protein amount and transport activity of SGLT1 in the BBM were similar to that in wild-type mice after gavage with glucose. In $R s 1^{-/-}$mice, no increase of SGLT1 amount and activity in the BBM was observed after gavage with D-glucose (Fig. 8, A and B). After gavage of wildtype mice with D-glucose, some upregulation of GLUT2 in the BBM was observed, as described earlier (Gouyon et al., 2003; Gorboulev et al., 2012) (Fig. 8C). In $R s 1^{-/-}$mice, the amount of GLUT2 in the BBM and upregulation of GLUT2 after gavage with $\mathrm{D}$-glucose were similar to that in wild-type mice (Fig. 8C). To estimate the velocity of the RS1-dependent glucose-induced upregulation of SGLT1 in the BBM, we incubated everted jejunum of wild-type and $R s 1^{-/-}$mice for 2 minutes without and with $2 \mathrm{mM} \mathrm{AMG}$ and isolated BBM vesicles. We measured phlorizin-inhibited AMG uptake into the vesicles and determined SGLT1 protein in the BBM after immunostaining (Fig. 8, D and E). Similar results were obtained to those obtained after glucose gavage, indicating that the glucose-dependent upregulation of SGLT1 occurs within 2 minutes. These data indicate that RS1 is critically involved in glucose-dependent upregulation of SGLT1 in the small intestine. We suggest the mechanism depicted in Fig. 9, A and B.

Differential Downregulation of AMG and Uridine Uptake by RS1-Reg Mutants in the Murine Small Intestine. We investigated whether selective downregulation of exocytotic pathways of hSGLT1 versus hCNT1 by RS1Reg mutants can be demonstrated in the mouse small intestine. The effects of mRS1-Reg(S19A) and mRS1-Reg (S19E) on phlorizin-inhibited AMG uptake and on sodiumdependent uridine uptake into enterocytes were measured. To protect the peptides from enzymatic degradation and to allow peptide entrance into the mucus, we coupled the mRS1-Reg mutants via disulfide linkage into biocompatible NG (Singh et al., 2013). The NG we used is a colloidal hydrophilic polymer network that is cross-linked by disulfides and additionally modified by the cell-penetrating peptide TAT (Torchilin, 2008). Under acidic conditions such as in the stomach, the NG is stable and the enclosed peptides are sterically protected from degradation. In basic milieu, like in the intestine, the NG becomes mucoadhesive from $\mathrm{OH}^{-}$-induced disulfide shuffling with the mucus layer (Bernkop-Schnurch, 2005), facilitating 


\section{Gavage with glucose}

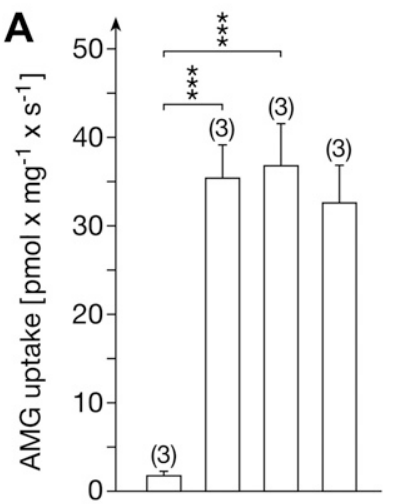

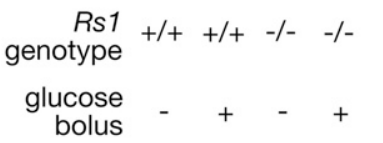

B

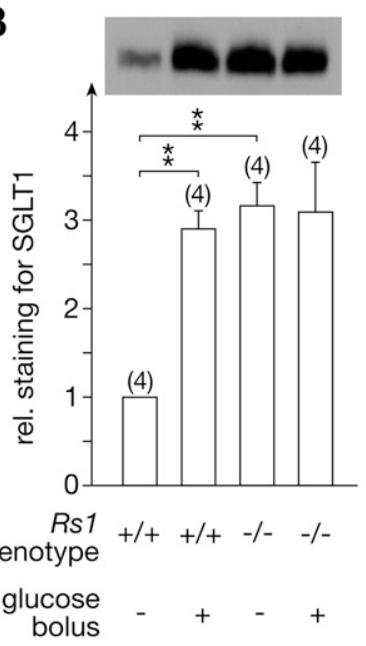

C
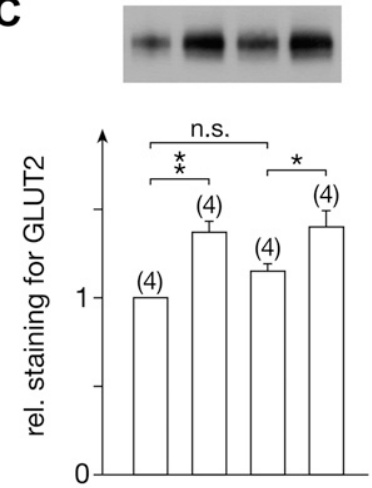

$\underset{\text { genotype }}{R s}+/++/+-/-\quad-/-$

glucose

bolus
Fig. 8. Effects of glucose on SGLT1 in the $\mathrm{BBM}$ of small intestine of wild-type mice and mice without RS1. (A-C) $R s 1^{+/+}$and $R s 1^{-1-}$ mice that had been fasted overnight were gavaged with $200 \mu \mathrm{l}$ water without or with D-glucose $(6 \mathrm{mg} / \mathrm{g}$ body weight). Thirty minutes later, the mice were sacrificed, the jejunum removed, and $\mathrm{BBM}$ vesicles were isolated. $(\mathrm{D}, \mathrm{E}) R s 1^{+/+}$ and $R S 1^{-1-}$ mice fasted overnight were sacrificed, and the jejunum was removed, washed with PBS, and everted. The everted jejunum was incubated for 2 minutes in PBS without or with $2 \mathrm{mM} \mathrm{AMG}$, and $\mathrm{BBM}$ vesicles were isolated. (A, D) Uptake of $100 \mu \mathrm{M}$ AMG was measured in the absence or presence of $0.2 \mathrm{mM}$ phlorizin, and phlorizin-inhibited AMG uptake was calculated. (B, E) Western blots of the $B B M$ vesicles were stained with a specific antibody against SGLT1, and staining was quantified by densitometry. (C) Western blots of BBM vesicles were stained with antibody against GLUT2, and staining was quantified by densitometry. Mean values \pm S.E. are shown and the number of experiments is indicated in parentheses. Significances of differences were calculated by ANOVA with post hoc Tukey comparison. $* P<0.05$; $* * P<0.01$; $* * * P<0.001$. contact of NG and bound peptides with the plasma membrane of the enterocytes. To compare the effects of the mRS1-Reg mutants on AMG uptake versus uridine uptake, we used $R s 1^{-1-}$ mice to avoid interference of regulation by endogenous RS1 (Fig. 10, A and B). Mice underwent gavage with $200 \mu \mathrm{l}$ of empty NG (control) or NG loaded with $1 \mathrm{nmol}$ mRS1-Reg mutant. Three hours after gavage, phlorizin-inhibited uptake of $10 \mu \mathrm{M}$ AMG or sodium-dependent uptake of $1 \mu \mathrm{M}$ uridine was measured. Uptake of $10 \mu \mathrm{M}$ AMG was not altered after gavage with mRS1-Reg(S19A), but it was downregulated by $21 \%(P<0.01)$ after gavage with mRS1-Reg(S19E) (Fig. 10A). On the other hand, uptake of $1 \mu \mathrm{M}$ uridine was downregulated by $22 \%(P<0.01)$ by mRS1-Reg(S19A) but was not altered by mRS1-Reg(S19E) (Fig. 10B). These data suggest that phosphorylation of RS1-Reg induces differential regulation of SGLT1 versus CNT1 in the small intestine. Measuring phlorizininhibited uptake of $1 \mathrm{mM}$ AMG after gavage of $m R S 1^{-1-}$ mice with NG loaded with mRS1-Reg(S19E) (Fig. 10C), we observed a downregulation by $32 \%(P<0.001)$, which indicates that the rise of intracellular AMG concentration during uptake of $1 \mathrm{mM}$ AMG does not blunt downregulation by mRS1-Reg(S19E).
We also investigated the effect of mRS1-Reg(S19E) on phlorizin-inhibited uptake of $10 \mu \mathrm{M}$ and $1 \mathrm{mM}$ AMG into enterocytes of wild-type mice (Fig. 10, D and E). As a result of downregulation of mSGLT1 in the BBM by endogenous mRS1 at low intracellular glucose, no further inhibitory effect on the uptake of $10 \mu \mathrm{M}$ AMG was observed (Fig. 10D); however, because downregulation via endogenous $\mathrm{mRS} 1$ is blunted at high intracellular glucose (Fig. 9B), uptake of $1 \mathrm{mM}$ AMG uptake was downregulated by mRS1-Reg(S19E) $(P<0.001)$ (Figs. 9C and 10E).

\section{Discussion}

We describe an $\mathrm{NH}_{2}$-terminal domain of regulatory protein RS1 (RS1-Reg) that controls differential post-translational downregulation of SGLT1 and CNT1 in the plasma membrane. In accordance with previous data obtained with total hRS1 protein or a fragment of hRS1 protein (Veyhl et al., 2003, 2006, 2007; Errasti-Murugarren et al., 2012), we present evidence that RS1-Reg downregulates the exocytotic pathways of hSGLT1 and hCNT1. We showed that downregulation 


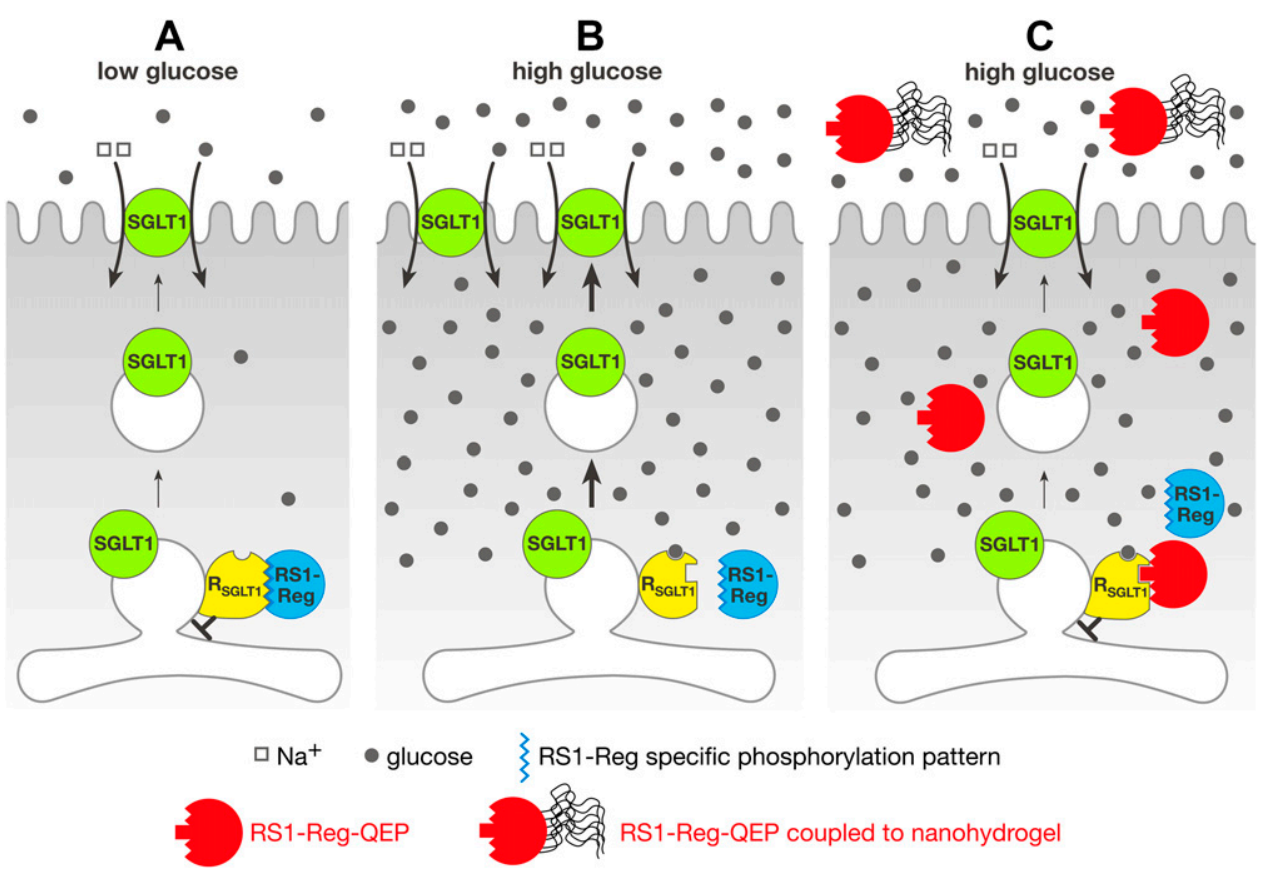

Fig. 9. Model describing the proposed role of RS1-Reg for upregulation of SGLT1 in small intestine after a glucose-rich meal and the effect of a RS1-Reg variant that blocks the glucose-induced upregulation. (A) When the concentration of glucose in the small intestinal lumen is low, RS1-Reg activates a receptor protein at the TGN $\left(\mathrm{R}_{(\mathrm{SGLT1})}\right)$, which inhibits the release of SGLT1-containing vesicles from the TGN. (B) After a glucose-rich meal, the intracellular glucose concentration increases and glucose binds to a lowaffinity glucose binding site at $\mathrm{R}_{\text {(SGLT1) }}$ or an associated protein. Glucose binding induces a conformational change of $\mathrm{R}_{\text {(SGLT1) }}$, which prevents RS1-Reg binding to $R_{\text {(SGLT1) }}$ and blunts blockage of the release of SGLT1-containing vesicles. (C) RS1-Reg variants in which serine in the $\mathrm{NH}_{2}$-terminal QSP motif is replaced by glutamate [RS1-Reg-QEP; e.g., hRS1$\operatorname{Reg}(\mathrm{S} 20 \mathrm{E})$ or mRS1-Reg(S19E)] bind to $\mathrm{R}_{\text {(SGLT1) }}$ in the presence of high intracellular glucose and induce blockage of SGLT1containing vesicles at high intracellular glucose. Coupling of RS1-Reg-QEP variants to NG induces uptake of RS1-Reg-QEP into enterocytes. of SGLT1 and CNT1 by RS1-Reg was not observed when the Golgi was dissociated with BFA and was prevented when fusion of intracellular vesicles with the plasma membrane was blocked by BTXB. This regulation is independent of endocytosis because we showed previously that the post-translational downregulation of SGLT1 by total RS1 protein was not influenced by inhibitors of endocytosis (Veyhl et al., 2006). Performing immunofluorescence laser scanning microscopy in LLC-PK 1 cells that express RS1 and SGLT1 endogenously, we observed that RS1 and SGLT1 are colocated with each other and with a TGN marker protein (Kroiss et al., 2006). Whereas RS1 was located all over the TGN, SGLT1 was localized in selective parts of the TGN. Within minutes after application of BFA, the Golgi was dissociated and SGLT1 and RS1 distributed into the cytosol (Kroiss et al., 2006). One hour after injection of total RS1 or RS1-Reg into oocytes expressing SGLT1 or CNT1, downregulation of about $50 \%$ of the transporters in the membrane was observed, which suggests that, in the absence of inhibitors of intracellular trafficking during this period, about half the SGLT1 or CNT1 molecules are retrieved from the plasma membrane and substituted via exocytotic pathways. On the basis of our present and previous data, we conclude that $\mathrm{RS} 1-\mathrm{Reg}$ regulates the release of different vesicle populations containing hSGLT1 or hCNT1 from TGN. In the present study, we also show that hRS1Reg-dependent downregulation of the exocytotic pathway of hSGLT1 was blunted when the intracellular glucose concentration was increased to $0.25 \mathrm{mM}$, whereas downregulation of the exocytotic pathway of hCNT1 was independent of glucose. Human RS1-Reg (hRS1-Reg) contains twice the QSP motif and the SDSDRIEP motif. Whereas hSGLT1 was downregulated by both peptides, hCNT1 was downregulated by SDSDRIEP but not by QSP. The determined low effective concentrations of RS1-Reg, QSP, and/or SDSDRIEP for downregulation of hSGLT1 and/or hCNT1 strongly suggest interactions with intracellular high-affinity receptor proteins. We interpret that the capability of hRS1-Reg to address two different exocytotic pathways selectively includes receptor proteins that recognize different modifications of RS1-Reg. These receptor proteins are supposed to activate the release of different vesicle populations from the TGN (see scheme in Fig. 11).

hRS1-Reg and RS1-Reg from mice (mRS1-Reg) contain many predicted phosphorylation sites and several predicted binding sites for regulatory proteins that are partially conserved between the two species. This indicates the possibility of a large diversity of modifications. To determine the role of phosphorylation on the interaction of RS1-Reg with the postulated receptor proteins, we investigated whether mimicking or preventing phosphorylation of RS1-Reg leads to differential effects on the affinities of the mutants for downregulation of hSGLT1 versus hCNT1. Because this turned out to be the case, we conclude that the selective release of different vesicle populations with different plasma membrane transporters from the TGN is controlled via differential phosphorylation of RS1-Reg (see scheme in Fig. 11).

We demonstrated different effects of RS1-Reg phosphorylation on regulation of two transporters. However, the more general interpretation that exocytotic pathways of various plasma membrane transporters are addressed by different regulatory states of RS1-Reg is considered warranted for several reasons. First, we observed that RS1 mediates posttranslational downregulation of most transporters that have been tested in addition to hSGLT1 and hCNT1. Posttranslational downregulation by RS1 was observed for human $\mathrm{Na}^{+}$-nucleoside cotransporters CNT1, CNT2, and CNT3 (Errasti-Murugarren et al., 2012), for organic cation transporter 1 (OCT1) from rat (Veyhl et al., 2003), and for OCT2 from rat and human (Reinhardt et al., 1999; Veyhl et al., 2003, 2006). Second, RS1-Reg contains many predicted phosphorylation sites and binding sites for regulatory proteins, thus suggesting a large diversity of modifications. Third, unlike downregulation of hSGLT1, which was blunted by high intracellular glucose, the downregulation of hOCT2 by hRS1 

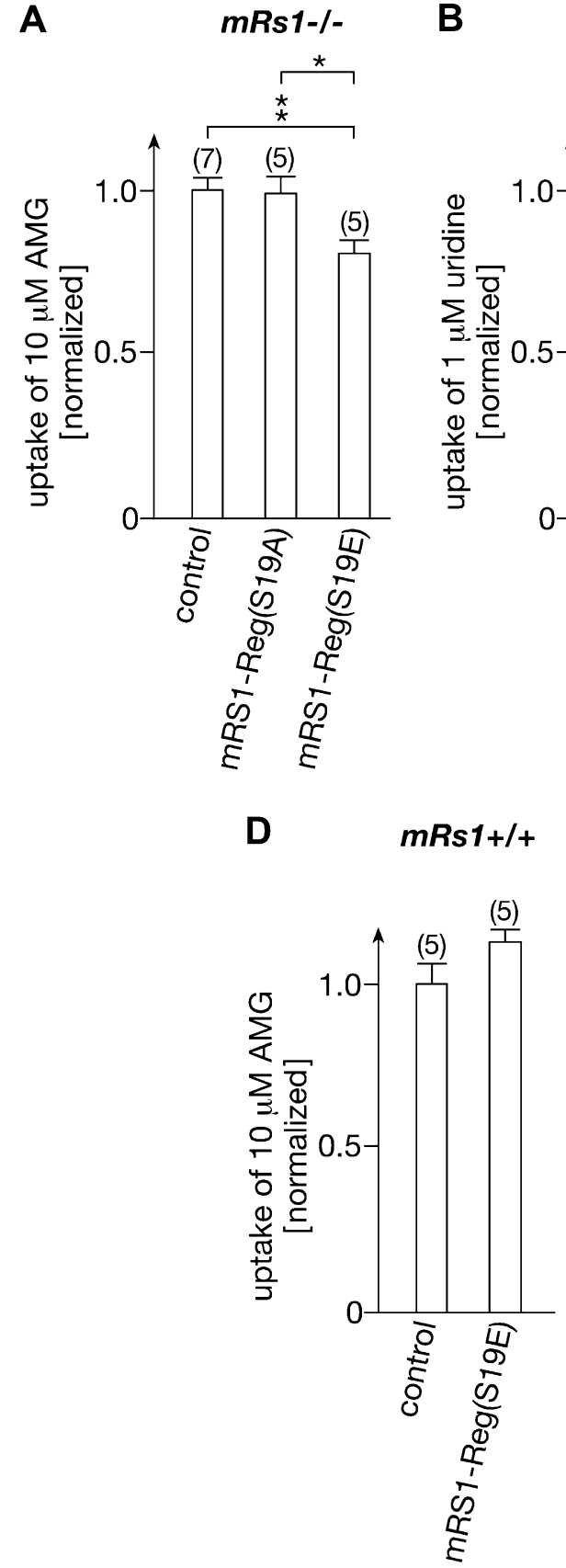

B mRs1-/-

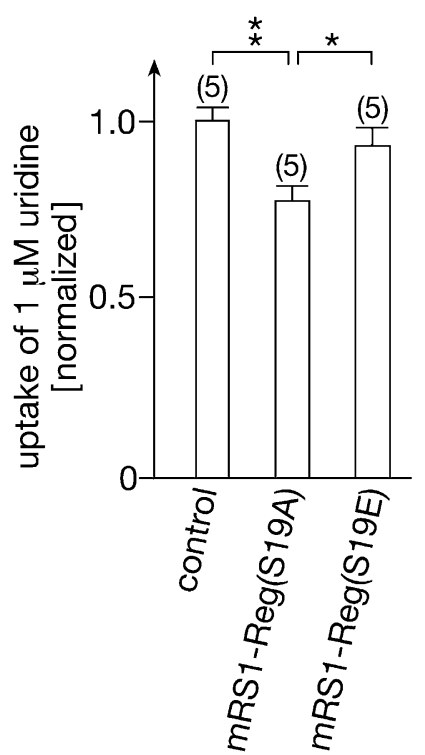

C

mRs1-/-



E $\quad m R s 1+/+$

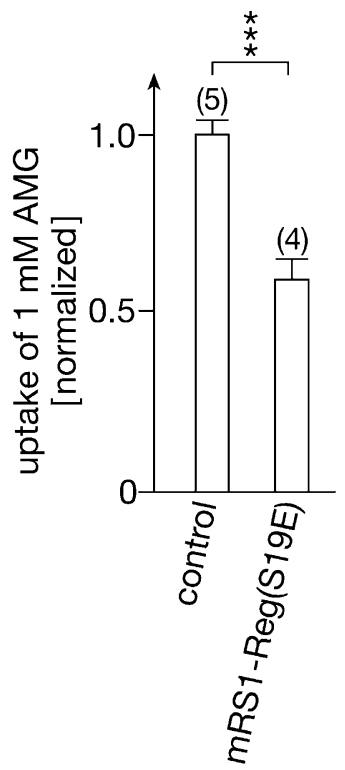

Fig. 10. Effects of gavage of mice with mRS1-Reg(S19E) and mRS1-Reg(S19A) on phlorizin inhibitable AMG uptake and sodium-dependent uridine uptake at low and high intracellular glucose. $R s 1^{-1-}$ or $R s 1^{+/+}$mice were gavaged with mRS1-Reg (S19A) or mRS1-Reg(S19E) coupled to NG or with unloaded NG (control). Three hours after gavage, phlorizin inhibited uptake of $10 \mu \mathrm{M}$ AMG (A and D, low intracellular glucose), sodium-dependent uptake of $1 \mu \mathrm{M}$ uridine (B), or phlorizininhibited uptake of $1 \mathrm{mM}$ AMG (C and E, high intracellular glucose) into enterocytes of segments of everted jejunum was measured. After gavage with unloaded NG, phlorizin-inhibited uptake of $10 \mu \mathrm{M}$ AMG was $35 \%$ higher in $R s 1^{-1-}$ mice compared with $R s 1^{+/+}$mice $(233 \pm 8.5$ vs. $173 \pm 11 \mathrm{pmol} \times \mathrm{cm}^{-1} \mathrm{~min}^{-1}$, mean \pm S.E.; $P<0.001$ for difference), whereas phlorizin inhibited uptake of $1 \mathrm{mM}$ AMG was $13 \%$ lower $(18.9 \pm 0.8$ vs. $21.6 \pm 0.9$ $\mathrm{nmol} \times \mathrm{cm}^{-1}$ min $^{-1}$, mean \pm S.E., $P<$ 0.05 for difference). Sodium-dependent uptake of $1 \mu \mathrm{M}$ uridine in $R s 1^{-1-}$ mice gavaged with unloaded NG was $55.7 \pm$ $2.1 \mathrm{pmol} \times \mathrm{cm}^{-1} \mathrm{~min}^{-1}$ (mean \pm S.E. $)$. (A-C) In $m R S 1^{-1-}$ mice, AMG uptake was downregulated by mRS1-Reg(S19E), whereas uridine uptake was downregulated by mRS1-Reg(S19A). (D, E) In $m R s 1^{+/+}$mice, uptake of $1 \mathrm{mM}$ AMG was downregulated by mRS1-Reg (S19E), whereas uptake of $10 \mu \mathrm{M}$ AMG was not altered. Uptake per $1 \mathrm{~cm}$ of jejunal segment is indicated. (mean \pm S.E.) The numbers of animals are indicated in parentheses. ${ }^{*} P<0.05 ; * * P<$ $0.01 ; * * * P<0.001$, ANOVA with posthoc Tukey comparison. was observed only in the presence of high intracellular glucose (Veyhl et al., 2006).

We provided in vivo evidence for differential regulation of endogenously expressed SGLT1 and CNT1 in the mouse small intestine. To exclude regulation by endogenous RS1, some of these experiments were performed in $R s 1^{-/-}$mice. We tested the effects of mRS1-Reg mutants preventing or mimicking phosphorylation of serine in the conserved QSP motif. To introduce the mutants into enterocytes, mice underwent gavage with NG-containing mRS1-Reg mutants coupled by disulfide linkage. Our data indicate that the NG represents an effective delivery system; however, mechanistic details remain to be resolved. It must be clarified whether the NG is dissociated within the glycocalix covering the enterocytes and whether released peptides or NG fragments bearing the peptides enter the cells by endocytosis. In addition, it must be determined how the peptides leave the endocytotic compartment. The mutations in the QSP motif of mRS1-Reg induced selective downregulation of phlorizin-inhibited AMG uptake mediated by mSGLT1 (Gorboulev et al., 2012) versus sodium-dependent uridine uptake mediated by mouse transporters CNT1 and CNT2 (Errasti-Murugarren et al., 2012).

We have provided evidence for the physiologic relevance of RS1-dependent downregulation of transporter abundance in the plasma membrane using SGLT1 as example. SGLT1 is the most abundant transporter in the BBM of small intestine and is rate limiting for glucose absorption (Gorboulev et al., 2012; Vrhovac et al., 2014; Wisniewski et al., 2014). After a glucoserich meal, the capacity of the small intestine for glucose absorption is increased by rapid upregulation of SGLT1 




Fig. 11. Scheme demonstrating how the differentially phosphorylated RS1-Reg domain of RS1 may interact with receptor proteins for SGLT1 $\left(R_{\text {(SGLT1) }}\right)$ or CNT1 $\left(R_{(\mathrm{CNT1})}\right)$ and may inhibit the release of vesicles containing SGLT1 or CNT1. It is hypothesized that $R_{(\text {SGLT1) }}$ ) and $\mathrm{R}_{\text {(CNT1) }}$ ) stimulate the release of vesicles from the TGN and that binding of $\mathrm{RS} 1$ to the receptor proteins prevents the stimulation of vesicle release, resulting in downregulation of the exocytotic pathways. Binding of glucose to $R_{(\text {SGLT1) }}$ is supposed to decrease binding of $R S 1$ to $R_{(\text {SGLT1) }}$ and thereby blunt RS1-mediated inhibition of vesicle release. Vesicular coat proteins and proteins that are directly involved in vesicle release are indicated in gray. RS1-Reg with different phosphorylation patterns is indicated in blue. Glucose and a proposed glucose binding site in $\mathrm{R}_{(\mathrm{SGLT1})}$ are indicated in red.

abundance in the BBM (Gorboulev et al., 2012). In the present report, we show that $\mathrm{RS} 1$ is critically involved in this glucosedependent short-term upregulation of SGLT1. Between meals, when the glucose concentration in the small intestine and enterocytes is low, RS1 downregulates the delivery of SGLT1 from the TGN to the plasma membrane (Fig. 9A). After the increase in glucose levels in the small intestine, however, RS1-mediated downregulation of SGLT1 delivery to the BBM is blunted (Fig. 9B). Under this condition, glucose may bind to the RS1-Reg receptor protein $\mathrm{R}_{(\mathrm{SGLT} 1)}$ (or to a protein associated with the receptor) and may induce dissociation of RS1Reg from the receptor protein, which may result in a disinhibition of receptor-mediated increase of vesicle release from the TGN, leading to upregulation of SGLT1 in the BBM (see scheme in Fig. 11).

The detection of a protein that steers glucose-dependent upregulation of glucose absorption provides a new target for the treatment of type 2 diabetes. If upregulation of SGLT1 in the small intestine after glucose-rich meals is prevented, secretion of the antidiabetic enterohormone glucagon-like peptide 1 (GLP-1) should be increased as observed after inhibition of SGLT1 and after impairment of glucose absorption by duodenal-jejunal bypass surgery (Jurowich et al., 2013; Powell et al., 2013). Decreased glucose absorption in the jejunum leads to increased GLP-1 secretion because the glucose concentration in the ileum is increased. L-cells, which are located in the distal ileum, secrete GLP-1 in response to glucose and short-chain fatty acids. Reversible inhibitors of
SGLT1 are tested for treatment of type 2 diabetes (Zambrowicz et al., 2012; Powell et al., 2013). Because these inhibitors may reach the systemic blood and SGLT1 is expressed in heart capillaries (Vrhovac et al., 2014), side effects on the cardiovascular system are anticipated. Blunting of SGLT1 upregulation in jejunum after glucose-rich meals by orally applied RS1-Reg derived peptides (e.g., $\mathrm{NH}_{2}$-terminal fragments of hRS1-Reg(S20E)) (Fig. 10C) may be a more save alternative to increase GLP-1 secretion because peptides may not enter the systemic blood.

We hope our report will stimulate investigations on different exocytotic pathways that are addressed by RS1-Reg. This includes the identification of RS1-Reg receptor proteins that mediate regulation of individual transporters, the characterization of RS1-Reg modifications that address the different receptors, and the characterization of vesicle populations carrying different plasma membrane transporters. Because the described regulation implicates more complex selective sorting, as has been described and expected (Anitei and Hoflack, 2011; Mayinger, 2011), a detailed investigation concerning the sorting of plasma membrane transporters at the TGN is warranted. In addition, the mechanism of how vesicle release is steered by RS1-Reg and activated by RS1Reg receptors must be elucidated. Recently, we obtained data indicating that ornithine decarboxylase (ODC) is the RS1-Reg receptor protein that activates downregulation of SGLT1 (C. Chintalapthi, T. Keller, T. Müller, V. Gorboulev, M. VeyhlWichmann and H. Koepsell, unpublished data). We observed that inhibition of ODC activity with the specific inhibitor difluoromethylornithine downregulates the exocytotic pathway of SGLT1 at the TGN, that RS1-Reg binds to ODC, that a hRS1Reg derived peptide inhibits ODC activity, and that downregulation of hSGLT1 by this peptide was blunted when enzymatic activity of ODC was blocked. These data suggest that polyamines generated by ODC stimulate release of SGLT1-containing vesicles from the TGN and that binding of RS1 to ODC inhibits the release of vesicles by blocking enzymatic activity of ODC. Although many questions remain unresolved, our data have broadened the view concerning the understanding of transporter regulation and opened new possibilities for investigating th regulation of cellular uptake.

\section{Acknowledgments}

The authors thank H. Walles (Department of Tissue Engineering and Regenerative Medicine, University Hospital Würzburg) and M. Lohse (Institute of Pharmacology and Toxicology, University Würzburg) for providing laboratory space and giving technical support and B.-C. Burckhardt and G. Burckhardt (Institute of Physiology and Pathophysiology, University Göttingen) for technical support.

\section{Authorship Contributions}

Participated in research design: Veyhl-Wichmann, Friedrich, Vernaleken, Pastor-Anglada, Groll, Koepsell.

Conducted experiments: Veyhl-Wichmann, Friedrich, Vernaleken, Singh, Kipp, Chintalapati.

Contributed new reagents or analytic tools: Singh, Gorboulev, Keller, Pipkorn, Pastor-Anglada, Groll, Koepsell.

Performed data analysis: Veyhl-Wichmann, Friedrich, Vernaleken, Kipp, Koepsell.

Wrote or contributed to the writing of the manuscript: Koepsell.

\section{References}

Anitei M and Hoflack B (2011) Exit from the trans-Golgi network: from molecules to mechanisms. Curr Opin Cell Biol 23:443-451. 
Bernkop-Schnürch A (2005) Thiomers: a new generation of mucoadhesive polymers. Adv Drug Deliv Rev 57:1569-1582.

Busch AE, Quester S, Ulzheimer JC, Waldegger S, Gorboulev V, Arndt P, Lang F, and Koepsell H (1996) Electrogenic properties and substrate specificity of the polyspecific rat cation transporter rOCT1. J Biol Chem 271:32599-32604.

Errasti-Murugarren E, Fernández-Calotti P, Veyhl-Wichmann M, Diepold M, PinillaMacua I, Pérez-Torras S, Kipp H, Koepsell H, and Pastor-Anglada M (2012) Role of the transporter regulator protein (RS1) in the modulation of concentrative nucleoside transporters (CNTs) in epithelia. Mol Pharmacol 82:59-67.

Filatova A, Leyerer M, Gorboulev V, Chintalapati C, Reinders Y, Müller TD, Srinivasan A, Hübner S, and Koepsell H (2009) Novel shuttling domain in a regulator (RSC1A1) of transporter SGLT1 steers cell cycle-dependent nuclear location. Traffic 10:1599-1618.

Gorboulev V, Schürmann A, Vallon V, Kipp H, Jaschke A, Klessen D, Friedrich A Scherneck S, Rieg T, and Cunard R, et al. (2012) Na-D-glucose cotransporter SGLT1 is pivotal for intestinal glucose absorption and glucose-dependent incretin secretion. Diabetes 61:187-196.

Gouyon F, Caillaud L, Carrière V, Klein C, Dalet V, Citadelle D, Kellett GL, Thorens B, Leturque A, and Brot-Laroche E (2003) Simple-sugar meals target GLUT2 at enterocyte apical membranes to improve sugar absorption: a study in GLUT2-null mice. $J$ Physiol 552:823-832

Groll J, Singh S, Albrecht K, and Moeller M (2009) Biocompatible and degradable nanogels via oxidation reactions of synthetic thiomers in inverse miniemulsion. $J$ Polym Sci A Polym Chem 47:5543-5549.

Gründemann D and Koepsell H (1994) Ethidium bromide staining during denaturation with glyoxal for sensitive detection of RNA in agarose gel electrophoresis. Anal Biochem 216:459-461.

Hediger MA and Rhoads DB (1994) Molecular physiology of sodium-glucose cotransporters. Physiol Rev 74:993-1026.

Ho SN, Hunt HD, Horton RM, Pullen JK, and Pease LR (1989) Site-directed mutagenesis by overlap extension using the polymerase chain reaction. Gene 77:51-59. Hopfer U, Nelson K, Perrotto J, and Isselbacher KJ (1973) Glucose transport in isolated brush border membrane from rat small intestine. J Biol Chem 248:25-32.

Jurowich CF, Rikkala PR, Thalheimer A, Wichelmann C, Seyfried F, Sander V, Kreissl M, Germer CT, Koepsell H, and Otto C (2013) Duodenal-jejunal bypass improves glycemia and decreases SGLT1-mediated glucose absorption in rats with streptozotocin-induced type 2 diabetes. Ann Surg 258:89-97.

Keller T, Elfeber M, Gorboulev V, Reiländer H, and Koepsell H (2005) Purification and functional reconstitution of the rat organic cation transporter OCT1. Biochemistry 44:12253-12263.

Köck K, Koenen A, Giese B, Fraunholz M, May K, Siegmund W, Hammer E, Völker U, Jedlitschky G, and Kroemer HK, et al. (2010) Rapid modulation of the organic anion transporting polypeptide 2B1 (OATP2B1, SLCO2B1) function by protein kinase C-mediated internalization. J Biol Chem 285:11336-11347.

Korn T, Kühlkamp T, Track C, Schatz I, Baumgarten K, Gorboulev V, and Koepsell H (2001) The plasma membrane-associated protein RS1 decreases transcription of the transporter SGLT1 in confluent LLC-PK 1 cells. J Biol Chem 276:45330-45340.

Kroiss M, Leyerer M, Gorboulev V, Kühlkamp T, Kipp H, and Koepsell H (2006) Transporter regulator RS1 (RSC1A1) coats the trans-Golgi network and migrates into the nucleus. Am J Physiol Renal Physiol 291:F1201-F1212.

Lambotte S, Veyhl M, Köhler M, Morrison-Shetlar AI, Kinne RKH, Schmid M, and Koepsell H (1996) The human gene of a protein that modifies Na-D-glucose cotransport. DNA Cell Biol 15:769-777.

Leto D and Saltiel AR (2012) Regulation of glucose transport by insulin: traffic control of GLUT4. Nat Rev Mol Cell Biol 13:383-396.

Mayinger P (2011) Signaling at the Golgi. Cold Spring Harb Perspect Biol 3:a005314

Mühlfeld S, Domanova O, Berlage T, Stross C, Helmer A, Keitel V, Häussinger D, and Kubitz R (2012) Short-term feedback regulation of bile salt uptake by bile salts in rodent liver. Hepatology 56:2387-2397.

Osswald C, Baumgarten K, Stümpel F, Gorboulev V, Akimjanova M, Knobeloch K-P, Horak I, Kluge R, Joost H-G, and Koepsell H (2005) Mice without the regulator gene $R s c 1 A 1$ exhibit increased $\mathrm{Na}^{+}$-D-glucose cotransport in small intestine and develop obesity. Mol Cell Biol 25:78-87.

Pipkorn R, Boenke C, Gehrke M, and Hoffmann R (2002) High-throughput peptide synthesis and peptide purification strategy at the low micromol-scale using the 96 well format. J Pept Res 59:105-114.
Powell DR, Smith M, Greer J, Harris A, Zhao S, DaCosta C, Mseeh F, Shadoan MK, Sands A, and Zambrowicz B et al. (2013) LX4211 increases serum glucagon-like peptide 1 and peptide YY levels by reducing sodium/glucose cotransporter 1 (SGLT1)-mediated absorption of intestinal glucose. J Pharmacol Exp Ther 345: 250-259.

Reinhardt J, Veyhl M, Wagner K, Gambaryan S, Dekel C, Akhoundova A, Korn T, and Koepsell H (1999) Cloning and characterization of the transport modifier RS1 from rabbit which was previously assumed to be specific for $\mathrm{Na}^{+}-\mathrm{D}$-glucose cotransport. Biochim Biophys Acta 1417:131-143.

Singh S, Zilkowski I, Ewald A, Maurell-Lopez T, Albrecht K, Möller M, and Groll J (2013) Mild oxidation of thiofunctional polymers to cytocompatible and stimulisensitive hydrogels and nanogels. Macromol Biosci 13:470-482.

Sumi M, Kiuchi K, Ishikawa T, Ishii A, Hagiwara M, Nagatsu T, and Hidaka H (1991) The newly synthesized selective $\mathrm{Ca}^{2+} /$ calmodulin dependent protein kinase II inhibitor KN-93 reduces dopamine contents in PC12h cells. Biochem Biophys Res Commun 181:968-975.

Torchilin VP (2008) Tat peptide-mediated intracellular delivery of pharmaceutical nanocarriers. Adv Drug Deliv Rev 60:548-558.

Valentin M, Kühlkamp T, Wagner K, Krohne G, Arndt P, Baumgarten K, Weber W, Segal A, Veyhl M, and Koepsell H (2000) The transport modifier RS1 is localized at the inner side of the plasma membrane and changes membrane capacitance. Biochim Biophys Acta 1468:367-380.

Vernaleken A, Veyhl M, Gorboulev V, Kottra G, Palm D, Burckhardt B-C, Burckhardt G, Pipkorn R, Beier N, and van Amsterdam C et al. (2007) Tripeptides of RS1 (RSC1A1) inhibit a monosaccharide-dependent exocytotic pathway of $\mathrm{Na}^{+}$-D glucose cotransporter SGLT1 with high affinity. J Biol Chem 282:28501-28513.

Veyhl M, Keller T, Gorboulev V, Vernaleken A, and Koepsell H (2006) RS1 (RSC1A1) regulates the exocytotic pathway of $\mathrm{Na}^{+}$-D-glucose cotransporter SGLT1. Am J Physiol Renal Physiol 291:F1213-F1223.

Veyhl M, Spangenberg J, Püschel B, Poppe R, Dekel C, Fritzsch G, Haase W, and Koepsell H (1993) Cloning of a membrane-associated protein which modifies activity and properties of the Na-D-glucose cotransporter. J Biol Chem 268 25041-25053.

Veyhl M, Wagner CA, Gorboulev V, Schmitt BM, Lang F, and Koepsell H (2003) Downregulation of the $\left.\mathrm{Na}^{+}\right)$- D-glucose cotransporter SGLT1 by protein RS1 (RSC1A1) is dependent on dynamin and protein kinase C. J Membr Biol 196: 71-81.

Vina-Vilaseca A, Bender-Sigel J, Sorkina T, Closs EI, and Sorkin A (2011) Protein kinase C-dependent ubiquitination and clathrin-mediated endocytosis of the cationic amino acid transporter CAT-1. J Biol Chem 286:8697-8706.

Vrhovac I, Balen ED, Klessen D, Burger C, Breljak D, Kraus O, Radović N, Jadrijević $\mathrm{S}$, Aleksic I, and Walles $\mathrm{T}$ et al. (2015) Localizations of $\mathrm{Na}^{+}$-D-glucose cotransporters SGLT1 and SGLT2 in human kidney and of SGLT1 in human small intestine, liver, lung, and heart. Pflugers Arch 467(9):1881-1898.

Wiśniewski JR, Friedrich A, Keller T, Mann M, and Koepsell H (2014) The impact of high fat diet on metabolism and immune defense in small intestine mucosa. $J$ Proteome Res 14(1):353-365.

Zambrowicz B, Freiman J, Brown PM, Frazier KS, Turnage A, Bronner J, Ruff D Shadoan M, Banks P, and Mseeh F et al. (2012) LX4211, a dual SGLT1/SGLT2 inhibitor, improved glycemic control in patients with type 2 diabetes in a randomized, placebo-controlled trial. Clin Pharmacol Ther 92:158-169.

Zeuthen T, Zeuthen E, and Klaerke DA (2002) Mobility of ions, sugar, and water in the cytoplasm of Xenopus oocytes expressing Na-coupled sugar transporters (SGLT1). J Physiol 542:71-87.

Zhang Q, Hong M, Duan P, Pan Z, Ma J, and You G (2008) Organic anion transporter OAT1 undergoes constitutive and protein kinase C-regulated trafficking through a dynamin- and clathrin-dependent pathway. J Biol Chem 283:32570-32579.

Zhang Q, Li S, Patterson C, and You G (2013) Lysine 48-linked polyubiquitination of organic anion transporter-1 is essential for its protein kinase C-regulated endocytosis. Mol Pharmacol 83:217-224.

Address correspondence to: Hermann Koepsell, Department of Molecular Plant Physiology and Biophysics, Julius-von-Sachs-Institute, Julius-vonSachs-Platz 2, 97082 Würzburg, Germany. E-mail: Hermann@koepsell.de 